\title{
RANDOM PERMUTATIONS WITHOUT MACROSCOPIC CYCLES
}

\author{
VOLKER BETZ, HELGE SCHÄFER, AND DIRK ZEINDLER
}

\begin{abstract}
We consider uniform random permutations of length $n$ conditioned to have no cycle longer than $n^{\beta}$ with $0<\beta<1$, in the limit of large $n$. Since in unconstrained uniform random permutations most of the indices are in cycles of macroscopic length, this is a singular conditioning in the limit. Nevertheless, we obtain a fairly complete picture about the cycle number distribution at various lengths. Depending on the scale at which cycle numbers are studied, our results include Poisson convergence, a central limit theorem, a shape theorem and two different functional central limit theorems.
\end{abstract}

\section{INTRODUCTION}

Uniform random permutations are among the oldest and best understood models of probability theory. One of their most prominent properties is that almost all indices are in macroscopic cycles: for all $\varepsilon>0$, the probability that a given index of a uniform permutation of length $n$ is in a cycle of length less than $n \varepsilon$ converges to $\varepsilon$ as $n \rightarrow \infty$. Classical results about uniform random permutations include the convergence of the renormalized cycle structure towards a Poisson-Dirichlet distribution [19, 24], convergence of joint cycle numbers towards independent Poisson random variables in total variation distance [3, and a central limit theorem for cumulative cycle numbers 14 .

Going beyond uniform random permutations, natural models are those where the probability measure is still invariant under conjugation with a transposition, i.e. it depends only on the cycle structure. One variety of such models are those with cycle weights, including the Ewens model [17] with applications in genetics, or more general cycle weight models 9, 11, 15, 16 with applications in quantum many body systems 7, , 8. Another variant is to condition on the absence of cycles of a given length. When the set $A \subset \mathbb{N}$ of forbidden cycle lengths is independent of the permutation length $n$, this goes under the name of $A$-permutations [26, 27. The case where the forbidden set of cycle lengths depends on $n$ is less well understood. We treat a fairly natural class of such models in the present paper.

Let $S_{n}$ denote the group of permutations of length $n$, and $\mathbb{P}_{n}$ the uniform probability measure on $S_{n}$. Each $\sigma \in S_{n}$ can be decomposed into disjoint cycles, and for $m \leq n$ we write $C_{m}=C_{m}(\sigma)$ for the number of cycles of $\sigma$ that have length $m$. For any sequence $\alpha=(\alpha(n))_{n \in \mathbb{N}}$ of nonnegative integers, let us write

$$
S_{n, \alpha}=\left\{\sigma \in S_{n}: C_{m}=0 \text { for all } m>\alpha(n)\right\}
$$

for the set of permutations having no cycle longer than $\alpha(n)$. The object of our study will be the (joint) distribution of the random variables $\left(C_{m}\right)_{m \leq \alpha(n)}$ under the uniform probability measure $\mathbb{P}_{n, \alpha}$ on $S_{n, \alpha}$.

All of our results hold under the following global assumption: We demand that there exist $a_{1}, a_{2} \in$ $(0,1)$, such that

$$
n^{a_{1}} \leq \alpha(n) \leq n^{a_{2}} .
$$

Under this condition, we find the following picture as $n \rightarrow \infty$ : cycles much shorter than $\alpha(n) / \log n$ behave under $\mathbb{P}_{n, \alpha}$ just like they would under $\mathbb{P}_{n}$, in the sense that their joint distribution converges, in total variation distance, to a suitable sequence of independent Poisson random variables; see Theorem 2.2. So in this regime, the conditioning that distinguishes $\mathbb{P}_{n, \alpha}$ from $\mathbb{P}_{n}$ has (asymptotically for large $n$ ) no effect on the distribution of cycle lengths. This effect starts to emerge when we study cycles with length of order $\alpha(n) / \log n$. We can see this quite explicitly for the special case $\alpha(n)=n^{\beta}$ with $0<\beta<1$ (see Section 2.2), and more generally in Theorem 2.3 . Finally, for cycles with length $c \alpha(n), 0 \leq c<1$, the influence of the restriction becomes clearly

2010 Mathematics Subject Classification. 60F17, 60F05, $60 \mathrm{C05.}$

Key words and phrases. random permutation, cycle structure, cycle weights, functional limit theorem, limit shape. 
visible; for instance, if $\alpha(n)=o(\sqrt{n})$, the number of cycles of length $c \alpha(n)$ is tending to infinity for $c$ sufficiently close to 1 and a central limit theorem holds, see Theorem 2.4. This behaviour is new and cannot be observed for classical random permutations.

A further striking difference between $\mathbb{P}_{n}$ and $\mathbb{P}_{n, \alpha}$ is that under the latter, asymptotically almost all cycles have size of order $\alpha(n)$. We make this precise in Theorem 2.5 where we find the relevant limit shape for the cumulative cycle numbers on this scale. Moreover, we find in Theorem 2.6 that the fluctuations around that limit shape satisfy a functional central limit theorem. Interestingly, the correct limiting process is Brownian bridge, and not the more usual Brownian motion that appears e.g. for unrestricted permutations at a suitable scaling, see [14] and equation (2.13).

The proofs of our results are based on the saddle point method of asymptotic analysis. In particular, we benefit from the precise estimates given by Manstavicius and Petuchovas [21] for the probability that an unconstrained permutation has no long cycles. While it is clear that such results must be useful for our purposes, it is surprising that they, and extensions of the methods by which they are proved, provide such a complete picture of the situation.

Let us give an outline of the paper. In Section 2, we state and discuss our results. Section 3 introduces the relevant saddle point method in our context and presents a general asymptotic equality which is at the base of almost all proofs of our main results. Section 4 then contains those proofs.

\section{MAin RESUlts}

2.1. Notation and standing assumptions. Let us recall that we require (1.1) for all of our results. Recall $S_{n}, S_{n, \alpha}, \mathbb{P}_{n}$ and $\mathbb{P}_{n, \alpha}$ from the introduction, and write $\mathbb{E}_{n}$ and $\mathbb{E}_{n, \alpha}$ for the expectations with respect to $\mathbb{P}_{n}$ and $\mathbb{P}_{n, \alpha}$, respectively. Recall also that $C_{m}=C_{m}(\sigma)$ denotes the number of cycles of length $m$ in the cycle decomposition of a permutation $\sigma$. The index $m$ will often depend on $n$ and $\alpha(n)$, but we sometimes omit this dependence when it is clear from the context.

When two sequences $\left(a_{n}\right)$ and $\left(b_{n}\right)$ are asymptotically equivalent, i.e. if $\lim _{n \rightarrow \infty} a_{n} / b_{n}=1$, we write $a_{n} \sim b_{n}$. We also use the usual $\mathcal{O}$ and $o$ notation, i.e. $f(n)=\mathcal{O}(g(n))$ means that there exists some constant $c>0$ so that $|f(n)| \leq c|g(n)|$ for large $n$, while $f(n)=o(g(n))$ means that for all $c>0$ there exists $n_{c} \in \mathbb{N}$ so that the inequality holds for all $n>n_{c}$.

2.2. Cycle counts. The most basic characteristics of the $C_{m}$ are their expected values. Let $x_{n, \alpha}$ be the unique positive solution of the equation

$$
n=\sum_{j=1}^{\alpha} x_{n, \alpha}^{j},
$$

and define

$$
\mu_{m}(n):=\frac{x_{n, \alpha}^{m}}{m} .
$$

Proposition 2.1. For all sequences $m=(m(n))_{n \in \mathbb{N}}$ with $m(n) \in \mathbb{N}$ and $m(n) \leq \alpha(n)$, we have

$$
\mathbb{E}_{n, \alpha(n)}\left[C_{m(n)}\right] \sim \mu_{m(n)}(n)
$$

as $n \rightarrow \infty$. Furthermore,

$$
\frac{1}{m} \log \left(m \mu_{m}\right)=\log x_{n, \alpha}=\frac{1}{\alpha}\left(\log \frac{n}{\alpha}+\log \log \frac{n}{\alpha}+\mathcal{O}\left(\frac{\log \log n}{\log n}\right)\right)
$$

for large $n$.

An example illustrates the amount of information that we can already extract from Proposition 2.1. Recall that for uniform permutations, $\mathbb{E}_{n}\left[C_{m}\right]=\frac{1}{m}$ for all $m \leq n[2$, Lemma 1.1]. We fix $\beta \in(0,1)$ and let $\alpha(n)=n^{\beta}$. Equation 2.3 then reads

$$
\log \left(m \mu_{m}\right)=m n^{-\beta}((1-\beta) \log n+\log \log n+\log (1-\beta)+o(1)) .
$$

We now have the following asymptotic regimes:

(1) For $m(n)=o\left(n^{\beta} / \log n\right)$, we have $\lim _{n \rightarrow \infty} \mu_{m(n)}(n) m(n)=1$. Thus we have

$$
\mathbb{E}_{n, \alpha(n)}\left[C_{m(n)}\right] \sim \frac{1}{m(n)}=\mathbb{E}_{n}\left[C_{m(n)}\right] .
$$


In particular, the limiting behavior is independent of $\beta$. We call this the classical regime.

(2) For $m(n)=y n^{\beta} / \log n$ with $y>0$, we get $\lim _{n \rightarrow \infty} \mu_{m(n)}(n) m(n)=\mathrm{e}^{y(1-\beta)}$. Thus

$$
\mathbb{E}_{n, \alpha(n)}\left[C_{m(n)}\right] \sim \frac{\mathrm{e}^{y(1-\beta)}}{m(n)}=\mathrm{e}^{y(1-\beta)} \mathbb{E}_{n}\left[C_{m(n)}\right]
$$

So in this regime, the number of cycles converges to zero more slowly than in unconstrained permutations. We therefore see that the constraint becomes visible in this region. Explicitly, we get

$$
\mu_{m(n)}(n) \sim \frac{\log n}{y n^{\beta}} \mathrm{e}^{y(1-\beta)} .
$$

The right-hand side above is minimal for $y=1 /(1-\beta)$ and then has the value $\mu_{m(n)}(n) \sim$ $\mathrm{e}(1-\beta) \frac{\log n}{n^{\beta}}$. Also, we have that $\mu_{m(n)}(n)$ is increasing as a function of $y$ for $y \geq 1 /(1-\beta)$.

(3) The next regime occurs when we put $m=c n^{\beta}$ for $0<c \leq 1$. Then

$$
\log \mu_{m}=(c(1-\beta)-\beta) \log n+c \log \log n+c \log (1-\beta)-\log c+o(1) .
$$

We see that $\mu_{m} \rightarrow 0$ when $c<\beta /(1-\beta)$, and $\mu_{m} \rightarrow \infty$ when $c \geq \beta /(1-\beta)$. So on this scale, the transition from finite cycle counts to infinite ones occurs. However, the case of infinite cycle counts can only occur if there exists $c \in(0,1]$ with $c \geq \beta /(1-\beta)$, which means that $\beta \leq 1 / 2$. This can be explained intuitively as follows: Since the maximal cycle length is $n^{\beta}$, a permutation $\sigma \in S_{n, \alpha}$ has (at least) $n / n^{\beta}=n^{1-\beta}$ cycles. If $\beta>1 / 2$ then $n^{\beta} \gg n^{1-\beta}$ and thus there are significantly more cycle lengths available than cycles. Therefore, for any given $\sigma \in S_{n, \alpha}, C_{m}(\sigma)$ must be equal to zero for the vast majority of $m \leq \alpha(n)$, and in absence of a convincing reason for concentration effects under $\mathbb{P}_{n, \alpha}$ we should expect to find $\mu_{m} \rightarrow 0$, as we indeed do.

The situation is reversed when $\beta<1 / 2$. We have in this case $n^{\beta} \ll n^{1-\beta}$, and thus there are always more cycles than available cycle lengths. The pigeon-hole principle now implies that at least $n^{1-\beta} / n^{\beta}=n^{1-2 \beta}$ cycles have the same length. Since $\beta<1 / 2$, we have $n^{1-2 \beta} \rightarrow \infty$ and thus $\max _{m} C_{m} \geq n^{1-2 \beta} \rightarrow \infty$. Note that Theorem 2.4 and equation $(2.15)$ below imply that in particular $C_{\alpha(n)} \rightarrow \infty$, except on a set with exponentially decaying probability.

We will now investigate the joint distributions of the random variables $C_{j}$. We start with the strongest result, which also has the most restrictive assumptions. Recall that the total variation distance of two probability measures $\mathbb{P}$ and $\tilde{\mathbb{P}}$ on a discrete probability space $\Omega$ is simply given by $\|\mathbb{P}-\tilde{\mathbb{P}}\|_{\mathrm{TV}}=\sum_{\omega \in \Omega}(\mathbb{P}(\omega)-\tilde{\mathbb{P}}(\omega))_{+}$.

Theorem 2.2. Let $b=(b(n))_{n}$ be a sequence of integers with $b(n)=o\left(\alpha(n)(\log n)^{-1}\right)$. Let $\mathbb{P}_{n, b(n), \alpha}$ be the distribution of $\left(C_{1}, \ldots, C_{b(n)}\right)$ under $\mathbb{P}_{n, \alpha}$, and let $\tilde{\mathbb{P}}_{b(n)}$ be the distribution of independent Poisson-distributed random variables $\left(Z_{1}, \ldots Z_{b(n)}\right)$ with $\tilde{\mathbb{E}}_{b(n)}\left(Z_{j}\right)=\frac{1}{j}$ for all $j \leq b(n)$. Then there exists $c<\infty$ so that for all $n \in \mathbb{N}$, we have

$$
\left\|\mathbb{P}_{n, b(n), \alpha}-\tilde{\mathbb{P}}_{b(n)}\right\|_{\mathrm{TV}} \leq c\left(\frac{\alpha(n)}{n}+b(n) \frac{\log n}{\alpha(n)}\right) .
$$

Let $\mathbb{P}_{n, b(n)}$ be the distribution of $\left(C_{1}, \ldots C_{b(n)}\right)$ under $\mathbb{P}_{n}$. Then it was proven by Arratia and Tavaré in [4, Theorem 2] that $\left\|\mathbb{P}_{n, b(n)}-\tilde{\mathbb{P}}_{b(n)}\right\|_{\mathrm{TV}} \rightarrow 0$ iff $b(n)=o(n)$. Thus the cycles of lengths $o\left(\alpha(n)(\log n)^{-1}\right)$ have a similar behaviour under $\mathbb{P}_{n}$ and under $\mathbb{P}_{n, \alpha}$. Furthermore, Arratia and Tavaré show in [3, Theorem 2] that there exists a function $F$ with $\log F(x) \sim-x \log x$ as $x \rightarrow \infty$ so that $\left\|\mathbb{P}_{n, b(n)}-\tilde{\mathbb{P}}_{b(n)}\right\|_{\mathrm{TV}} \leq F(n / b(n))$. Thus this total variation distance is converging to zero superexponentially fast as a function of $n / b(n)$. This fast decay rate is currently only known to occur for the uniform measure; all other known results (including ours, but see also see [25] for the Ewans measure) on convergence in total variation distance for measures of random permutations only come with algebraic decay rates.

We can slightly relax the condition $b(n)=o\left(\alpha(n)(\log n)^{-1}\right)$ in Theorem 2.2 if we only consider convergence of finite-dimensional distributions. What is more, we can in this case apply a 'tilt' as we would do in large deviations theory in order to get a better understanding of those cases where 
$\mu_{m} \rightarrow 0$ in Proposition 2.1. For $\nu \in \mathbb{R}_{0}^{+}$, consider the tilted cycle numbers $C_{k}^{(\nu)}$ with distribution

$$
\mathbb{P}\left[C_{m_{1}}^{\left(\nu_{1}\right)}=l_{1}, \ldots, C_{m_{k}}^{\left(\nu_{k}\right)}=l_{k}\right]=\frac{1}{Z}\left(\prod_{j=1}^{k} \frac{\mathrm{e}^{\nu_{j}}}{\nu_{j}^{l_{j}}}\right) \mathbb{P}_{n, \alpha}\left[C_{m_{1}}=l_{1}, \ldots, C_{m_{k}}=l_{k}\right]
$$

for all $l_{1}, \ldots, l_{k} \in \mathbb{N}_{0}$, where $Z$ is a normalizing constant.

Theorem 2.3. Let $\left(m_{1}(n)\right)_{n}, \ldots,\left(m_{k}(n)\right)_{n}$ be sequences of integers with $m_{k}(n) \leq \alpha(n)$ for all $n$ and $m_{i}(n) \neq m_{j}(n)$ for $i \neq j$. Assume that for all $j \leq k$,

$$
\limsup _{n \rightarrow \infty} \mu_{m_{j}(n)}(n)<\infty \text {. }
$$

Then, as $n \rightarrow \infty$,

$$
\left(C_{m_{1}}^{\left(\mu_{m_{1}}\right)}, \ldots, C_{m_{k}}^{\left(\mu_{m_{k}}\right)}\right) \stackrel{d}{\longrightarrow}\left(Z_{1}, \ldots, Z_{k}\right),
$$

where the $Z_{j}$ are independent Poisson distributed random variables with parameter 1.

From equation (2.3) and our assumptions on $\alpha(n)$ in (1.1), it follows that a sufficient condition for (2.6) is that $m_{j}(n) \leq c \alpha(n)$ for some $c<\frac{a_{1}}{1-a_{1}}$ with $a_{1}$ as in 1.1 . The case when $m_{j}(n)$ converges to a limit is already covered by Theorem 2.2. The most interesting applications of Theorem 2.3 are in the situation when $\mu_{m_{j}}$ converges to a limit while $m_{j} \rightarrow \infty$ as $n \rightarrow \infty$. For instance, if $\mu_{m} \rightarrow 0$, $C_{m}$ converges in distribution to the trivial Poisson distribution with parameter 0 , but just like it is the case in large deviations theory, the tilt allows us to extract much more information about this convergence. We have in particular that for all $j \in \mathbb{N}_{0}$, the probability $\mathbb{P}_{n, \alpha}\left[C_{m}=j\right]$ decays like $\mu_{m}^{-j}$.

We now treat the case of diverging expected cycle numbers. Here, the standard rescaling leads to a central limit theorem.

Theorem 2.4. Let $\left(m_{1}(n)\right)_{n}, \ldots,\left(m_{k}(n)\right)_{n}$ be sequences of integers with $m_{j}(n) \leq \alpha(n)$ for all $n$ and all $j$ and $m_{i}(n) \neq m_{j}(n)$ for $i \neq j$. Assume that $\mu_{m_{j}(n)}(n) \rightarrow \infty$ as $n \rightarrow \infty$ for all $j$. Assume finally that in (1.1), we have $a_{1}>1 / 7$. Define

$$
\widetilde{C}_{m_{j}}:=\frac{C_{m_{j}}-\mu_{m_{j}}}{\sqrt{\mu_{m_{j}}}} .
$$

Then

$$
\left(\widetilde{C}_{m_{1}}, \ldots, \widetilde{C}_{m_{k}}\right) \stackrel{d}{\longrightarrow}\left(N_{1}, \ldots, N_{k}\right) \quad \text { as } n \rightarrow \infty,
$$

where $\left(N_{j}\right)_{j=1}^{k}$ are independent, standard normal distributed random variables.

The condition $\alpha(n) \geq n^{\frac{1}{7}+\delta}$ is a technical one, and making it allows to avoid significant technical complications. A forthcoming paper will show that the theorem holds under condition (1.1).

\subsection{Cumulative cycle numbers. Let}

$$
K_{m}=\sum_{j=1}^{m} C_{j}
$$

be the number of cycles with lengths less than $m$. Since no cycle can be larger than $\alpha(n)$, the total number of cycles $K_{\alpha(n)}$ is at least $\geq n / \alpha(n)$. In [6] it is shown that $K_{\alpha(n)} \sim \frac{n}{\alpha(n)}$, and so the random variable $\frac{K_{m(n)}}{n / \alpha(n)}$ gives the fraction of cycles that have length up to $m(n)$. We now define

$$
b_{t}(n):=\max \left\{\alpha(n)+\left\lfloor\log (t) \frac{\alpha(n)}{\log \left(\frac{n}{\alpha(n)}\right)}\right\rfloor, 0\right\}, \quad 0 \leq t \leq 1 .
$$

We have the following limit shape of the random function $t \mapsto K_{b_{t}(n)}$ :

Theorem 2.5. We have for each $\epsilon>0$,

$$
\mathbb{P}_{n, \alpha}\left[\sup _{t \in[0,1]}\left|\frac{K_{b_{t}(n)}}{n / \alpha(n)}-t\right|>\epsilon\right] \rightarrow 0 \text { as } n \rightarrow \infty
$$


When we choose $t=1$ in Theorem 2.5, then $b_{t}(n)=\alpha(n)$ and we recover the result in [6]. Furthermore, if we define

$$
\nu_{\delta}:=\lim _{n \rightarrow \infty} \frac{K_{b_{\delta}(n)}}{K_{\alpha(n)}} \text { for } \delta>0,
$$

then $\nu_{\delta}$ can be interpreted as the limit as $n \rightarrow \infty$ of the fraction of cycles smaller than $b_{\delta}(n)$. Theorem 2.5 now shows that $\nu_{\delta} \rightarrow \delta$ for all $0<\delta \leq 1$. Since $b_{\delta}(n)=\alpha(n)(1+o(1))$ for all $\delta>0$, we immediately get that almost all cycles live in a scale of the form $\alpha(n)(1+o(1))$.

A theorem similar to Theorem 2.5 can be proved for the number of elements in the cycles with size smaller than $m$. If we set $S_{m}=\sum_{j=1}^{m} j C_{j}$, then trivially $S_{\alpha}=n$, and we can show that

$$
\mathbb{P}_{n, \alpha}\left[\sup _{t \in[0,1]}\left|\frac{S_{b_{t}(n)}}{n}-t\right|>\epsilon\right] \rightarrow 0 \text { as } n \rightarrow \infty .
$$

The proof, which is similar to the proof of Theorem 2.5, can be found in [23, Theorem 2.7.2]. In the next theorem we take a closer look at the fluctuations around the limit shape of $K_{b_{t}(n)}$.

Theorem 2.6. Let

$$
L_{t}(n):=\frac{K_{b_{t}(n)}-\sum_{j=1}^{b_{t}(n)} \frac{x_{n, \alpha}^{j}}{j}}{\sqrt{n / \alpha(n)}} .
$$

Then $\left(L_{t}(n)\right)_{t \in[0,1]}$ converges in distribution to the standard Brownian bridge in $\mathcal{D}[0,1]$, where $\mathcal{D}[0,1]$ is the space of cadlag functions on $[0,1]$, endowed with the Skorohod topology.

Remark 2.7. (1) As above, we can do the same construction for the indices instead of the cycles. With $S_{m}$ being as in the remark after Theorem 2.5, we have that

$$
\tilde{L}_{t}(n):=\frac{S_{b_{t}(n)}-\sum_{j=1}^{b_{t}(n)} x_{n, \alpha}^{j}}{\sqrt{n \alpha(n)}}
$$

converges to the Brownian bridge in $\mathcal{D}[0,1]$. The proof is similar to the one of Theorem 2.6, so we refer to [23, Theorem 2.7.6].

(2) When $t=1$ in Theorem 2.6 the variance of the limit is zero. However, it has been shown in [6] that there exists a different rescaling so that the Gaussian fluctuations persist in the limit: We have

$$
\frac{K_{\alpha(n)}-\sum_{j=1}^{\alpha(n) \frac{x_{n, \alpha}^{j}}{j}}}{\sqrt{\frac{n}{\alpha(n)(\log (n / \alpha(n)))^{2}}}} \stackrel{d}{\longrightarrow} \mathcal{N}(0,1) .
$$

Of course, no such statement can hold for $S_{\alpha(n)}$ since $S_{\alpha(n)}-\sum_{j=1}^{\alpha(n)} x_{n, \alpha}^{j}=S_{\alpha(n)}-n=0$.

(3) For unrestricted permutations, Delaurentis and Pittel [14] show that the stochastic process

$$
\left(\frac{\sum_{j=1}^{\left\lfloor n^{t}\right\rfloor} C_{j}-t \log (n)}{\sqrt{\log (n)}}\right)_{t \in[0,1]}
$$

converges in distribution to the Brownian motion in $[0,1]$. Interestingly, this holds for restricted permutations as well, and we have already shown it! Indeed, the convergence in total variation distance from Theorem 2.2 is strong enough to show that for all $t<a_{1}$ (cf. (1.1)), convergence to the Brownian motion also holds when the $C_{j}$ in 2.13 are those of constrained permutations. Hence, in the case of constrained permutations, we actually have two functional central limit theorems: one for 'short' cycles and one for the ones very close to the maximal cycle length.

(4) The asymptotic behaviour of the longest cycles in a random permutation is one of the most frequently asked questions and is in particular still open for random permutations with polynomially and logarithmically growing cycle weights. We denote by $\ell_{1}(\sigma)$ the length of the longest cycle in a permutation, $\ell_{2}(\sigma)$ the length of the second longest cycle in a permutation and so on. We have for each $k \in \mathbb{N}$

$$
\frac{1}{\alpha(n)}\left(\ell_{1}, \ell_{2}, \ldots, \ell_{k}\right) \stackrel{d}{\longrightarrow}(\underbrace{1,1, \ldots, 1}_{\mathrm{k} \text { times }}) .
$$


Further, if $\alpha(n)=\mathcal{O}\left(n^{1 / 2}\right)$ and $\alpha(n) \geq n^{\frac{1}{7}+\delta}$ for some $\delta>0$ then

$$
\mathbb{P}_{n, \alpha}\left[\left(\ell_{1}, \ell_{2}, \ldots, \ell_{k}\right) \neq(\alpha(n), \ldots, \alpha(n))\right] \rightarrow 0 \text { as } n \rightarrow \infty .
$$

These statements follow immediately from Theorems 2.4 and 2.5

\section{Generating functions And the SAdDle-Point Method}

Generating functions and their connection with analytic combinatorics form the backbone of the proofs in this paper. More precisely, we will determine formal generating functions for all relevant moment-generating functions and then use the saddle-point method to determine the asymptotic behaviour of these moment-generating functions as $n \rightarrow \infty$.

Let $\left(a_{n}\right)_{n \in \mathbb{N}}$ be a sequence of complex numbers. Then its ordinary generating function is defined as the formal power series

$$
f(z):=\sum_{n=0}^{\infty} a_{n} z^{n} .
$$

The sequence may be recovered by formally extracting the coefficients

$$
\left[z^{n}\right] f(z):=a_{n}
$$

for any $n$. The first step is now to consider a special case of Pólya's Enumeration Theorem, see [22, $\S 16$, p. 17], which connects permutations with a specific generating function.

Lemma 3.1. Let $\left(q_{j}\right)_{j \in \mathbb{N}}$ be a sequence of complex numbers. We then have the following identity between formal power series in $z$,

$$
\exp \left(\sum_{j=1}^{\infty} \frac{q_{j} z^{j}}{j}\right)=\sum_{k=0}^{\infty} \frac{z^{k}}{k !} \sum_{\sigma \in S_{k}} \prod_{j=1}^{k} q_{j}^{C_{j}}
$$

where $C_{j}=C_{j}(\sigma)$ are the cycle counts. If either of the series in (3.1) is absolutely convergent, then so is the other one.

Extracting the $n$th coefficient yields

$$
\left[z^{n}\right] \exp \left(\sum_{j=1}^{\infty} \frac{q_{j} z^{j}}{j}\right)=\frac{1}{n !} \sum_{\sigma \in S_{n}} \prod_{j=1}^{n} q_{j}^{C_{j}} .
$$

Setting $q_{j}=\mathbb{1}_{\{j \leq \alpha(n)\}}$ we obtain

$$
Z_{n, \alpha}:=\frac{\left|S_{n, \alpha}\right|}{n !}=\left[z^{n}\right] \exp \left(\sum_{j=1}^{\alpha} \frac{z^{j}}{j}\right) .
$$

For distinct integers $1 \leq m_{k} \leq \alpha(n), 1 \leq k \leq K$ with $k, K \in \mathbb{N}$ and $s_{1}, \ldots, s_{K} \in \mathbb{R}$, we obtain

$$
\mathbb{E}_{n, \alpha}\left[\mathrm{e}^{\sum_{k=1}^{K} s_{k} C_{m_{k}}}\right]=\frac{1}{Z_{n, \alpha}}\left[z^{n}\right] \exp \left(\sum_{k=1}^{K}\left(\mathrm{e}^{s_{k}}-1\right) \frac{z^{m_{k}}}{m_{k}}\right) \exp \left(\sum_{j=1}^{\alpha(n)} \frac{z^{j}}{j}\right) .
$$

This equation follows immediately from the definition of $\mathbb{E}_{n, \alpha}$ and 3.2 . Similarly, for $0=t_{0} \leq$ $t_{1}<\cdots<t_{m} \leq t_{m+1}=1$, we have

$$
\mathbb{E}_{n, \alpha}\left[\mathrm{e}^{\sum_{i=1}^{m} s_{i} K_{b_{t_{i}}}(n)}\right]=\frac{1}{Z_{n, \alpha}}\left[z^{n}\right] \exp \left(\sum_{i=0}^{m} \sum_{j=b_{t_{i}}(n)+1}^{b_{t_{i+1}}(n)} \frac{\mathrm{e}^{\sum_{\ell=i+1}^{m} s_{\ell}} z^{j}}{j}\right) .
$$

At this stage, all parameters can depend on the system size $n$. A way to extract the series coefficients from expressions such as (3.4) and (3.5) is the saddle point method, a standard tool in asymptotic analysis. The basic idea is to rewrite the expression 3.2 as a complex contour integral and choose the path of integration in a convenient way. The details of this procedure depend on the situation at hand and need to be done on a case by case basis. A general overview over the saddle-point method can be found in [18, page 551].

We now treat the most general case of the saddle point method that is relevant for the present situation. Let $\boldsymbol{q}=\left(q_{j, n}\right)_{1 \leq j \leq \alpha(n), n \in \mathbb{N}}$ be a triangular array. We assume that all $q_{j, n}$ are nonnegative, 
real numbers and that for each $n \in \mathbb{N}$ there exists a $j$ such that $q_{j, n}>0$. We then define $x_{n, \boldsymbol{q}}$ as the unique positive solution of

$$
n=\sum_{j=1}^{\alpha(n)} q_{j, n} x_{n, \boldsymbol{q}}^{j}
$$

Let further

$$
\lambda_{p, n}:=\lambda_{p, n, \alpha, \boldsymbol{q}}:=\sum_{j=1}^{\alpha(n)} q_{j, n} j^{p-1} x_{n, \boldsymbol{q}}^{j},
$$

where $p \geq 1$ is a natural number. Due to Equation (3.6),

$$
\lambda_{p, n} \leq n(\alpha(n))^{p-1}
$$

holds for all $p \geq 1$.

Let us write $a_{n} \approx b_{n}$ when there exist constants $c_{1}, c_{2}>0$ such that

$$
c_{1} b_{n} \leq a_{n} \leq c_{2} b_{n}
$$

for large $n$. We further say that

$$
f_{n}(t)=\mathcal{O}\left(g_{n}(t)\right) \text { uniformly in } t \in T_{n}
$$

if there are constants $c, N>0$ such that $\sup _{t \in T_{n}}\left\{\left|\frac{f_{n}(t)}{g_{n}(t)}\right|\right\} \leq c$ for all $n \geq N$.

We will call an array $\boldsymbol{q}$ admissible if the following three conditions are met:

(i): We have

$$
\alpha(n) \log x_{n, \boldsymbol{q}} \approx \log \frac{n}{\alpha(n)} .
$$

(ii): We have

$$
\lambda_{2, n} \approx n \alpha(n) .
$$

(iii): There exists a sequence $n \mapsto b(n)$ with $b(n) / \alpha(n)<(1-\delta)$ for some $\delta>0$, and such that $q_{j, n} \geq c>0$ for all $j \geq b(n)$ and some constant $c>0$.

Note that condition (i) implies in particular that $\lim _{n \rightarrow \infty} x_{n, \boldsymbol{q}}=1$.

Let $B_{r}(0)$ denote the circle with center 0 and radius $r$ in the complex plane. Let $\delta, K, N>0$. Given an array $\boldsymbol{q}$, we will call a sequence of complex-valued functions $f_{n}(\delta, K, N)$-admissible if the following three conditions are met:

(i): For all $n, f_{n}$ is holomorphic on $B_{x_{n, q}+\delta}(0)$.

(ii): For all $n \geq N$, we have

$$
\sup _{z \in \partial B_{x_{n}, \boldsymbol{q}}(0)}\left|f_{n}(z)\right| \leq n^{K}\left|f_{n}\left(x_{n, \boldsymbol{q}}\right)\right| .
$$

(iii) Let

$$
\theta_{n}:=n^{-\frac{5}{12}}(\alpha(n))^{-\frac{7}{12}}
$$

For

we have $\lim _{n \rightarrow \infty}\left\|f_{n}\right\|_{n}=0$.

$$
\left\|f_{n}\right\|_{n}:=\theta_{n} \sup _{|\theta| \leq \theta_{n}} \frac{\left|f_{n}^{\prime}\left(x_{n, \boldsymbol{q}} \mathrm{e}^{\mathrm{i} \theta}\right)\right|}{\left|f_{n}\left(x_{n, \boldsymbol{q}}\right)\right|}
$$

We refer to $\left(f_{n}\right)$ as admissible if there are $\delta, K, N>0$ such that $\left(f_{n}\right)$ is $(\delta, K, N)$-admissible. We are now in the position to formulate our general saddle point result.

Proposition 3.2. Let $\boldsymbol{q}$ be an admissible triangular array, and $\left(f_{n}\right)$ an admissible sequence of functions. Then, as $n \rightarrow \infty$,

$$
\left[z^{n}\right] f_{n}(z) \exp \left(\sum_{j=1}^{\alpha(n)} \frac{q_{j, n}}{j} z^{j}\right)=f_{n}\left(x_{n, \boldsymbol{q}}\right) \frac{\mathrm{e}^{\lambda_{0, n}}}{x_{n, \boldsymbol{q}}^{n} \sqrt{2 \pi \lambda_{2, n}}}\left(1+\mathcal{O}\left(\frac{\alpha(n)}{n}\right)\right)\left(1+\mathcal{O}\left(\left\|f_{n}\right\|_{n}\right)\right) .
$$

Moreover, if we fix $\delta, K, N>0$, then the error terms in (3.13) are uniform in all $(\delta, K, N)$ admissible sequences $\left(f_{n}\right)$. 
Proof. Let $\left(f_{n}\right)$ be $(\delta, K, N)$-admissible for some $\delta, K, N>0$. Cauchy's integral formula gives

$$
M_{n}:=\left[z^{n}\right] f_{n}(z) \exp \left(\sum_{j=1}^{\alpha(n)} \frac{q_{j, n}}{j} z^{j}\right)=\frac{1}{2 \pi \mathrm{i}} \int_{\partial B_{r}(0)} f_{n}(z) \exp \left(\sum_{j=1}^{\alpha(n)} \frac{q_{j, n}}{j} z^{j}\right) \frac{\mathrm{d} z}{z^{n+1}}
$$

for any $r$ such that $f_{n}$ is holomorphic on $B_{r}(0)$. Condition (i) on $f_{n}$ guarantees that we can take $r=x_{n, \boldsymbol{q}}$. We then rewrite

$$
M_{n}=\frac{1}{2 \pi x_{n, \boldsymbol{q}}^{n}} \int_{-\pi}^{\pi} f_{n}\left(x_{n, \boldsymbol{q}} \mathrm{e}^{\mathrm{i} \theta}\right) \exp \left(\sum_{j=1}^{\alpha(n)} \frac{q_{j, n}}{j}\left(x_{n, \boldsymbol{q}} \mathrm{e}^{\mathrm{i} \theta}\right)^{j}-\mathrm{i} n \theta\right) \mathrm{d} \theta .
$$

For the remainder of the proof, we will write $x$ instead of $x_{n, \boldsymbol{q}}$ and $\alpha$ instead of $\alpha(n)$ for lighter notation. We define

$$
g_{n}(\theta):=\sum_{j=1}^{\alpha} q_{j, n} \frac{\mathrm{e}^{\mathrm{i} j \theta}-1}{j} x^{j}-\mathrm{i} n \theta
$$

and obtain

$$
M_{n}=\frac{\exp \left(\sum_{j=1}^{\alpha} \frac{q_{j, n}}{j} x^{j}\right)}{2 \pi x^{n}} \int_{-\pi}^{\pi} f_{n}\left(x \mathrm{e}^{\mathrm{i} \theta}\right) \exp \left(g_{n}(\theta)\right) \mathrm{d} \theta .
$$

Note $g_{n}(0)=0$ and, by (3.6), $g_{n}^{\prime}(0)=0$. Similarly, $\left|g_{n}^{(p)}(\theta)\right| \leq \lambda_{p, n}$ and $g_{n}^{(p)}(0)=\mathrm{i}^{p} \lambda_{p, n}$ for $p \geq 2$. For $|\theta| \leq \theta_{n}$ (see (3.11), equation (3.7) implies that $\lambda_{p, n}|\theta|^{p} \leq(n / \alpha)^{1-5 p / 12}$. Therefore a Taylor expansion around 0 gives

$$
g_{n}(\theta)=-\frac{\lambda_{2, n}}{2} \theta^{2}-\mathrm{i} \frac{\lambda_{3, n}}{6} \theta^{3}+\mathcal{O}\left(\lambda_{4, n} \theta^{4}\right)
$$

and

$$
\exp \left(g_{n}(\theta)\right)=\exp \left(-\frac{\lambda_{2, n}}{2} \theta^{2}\right)\left(1-\mathrm{i} \frac{\lambda_{3, n}}{6} \theta^{3}+\mathcal{O}\left(\lambda_{3, n}^{2} \theta^{6}\right)\right)\left(1+\mathcal{O}\left(\lambda_{4, n} \theta^{4}\right)\right),
$$

where the error terms are uniform in $\theta \in\left[-\theta_{n}, \theta_{n}\right]$. As for $f_{n}$, we have

$$
f_{n}\left(x \mathrm{e}^{\mathrm{i} \theta}\right)=f_{n}(x)+\mathrm{i} \int_{0}^{\theta} f_{n}^{\prime}\left(x \mathrm{e}^{\mathrm{i} \varphi}\right) x \mathrm{e}^{\mathrm{i} \varphi} \mathrm{d} \varphi .
$$

Estimating the modulus of the integrand in the second term by its maximum and using assumption (3.12), we find that, uniformly in $\theta \in\left[-\theta_{n}, \theta_{n}\right]$,

$$
f_{n}\left(x \mathrm{e}^{\mathrm{i} \theta}\right)=f_{n}(x)\left(1+\mathcal{O}\left(\left\|f_{n}\right\|_{n}\right)\right) .
$$

Here, the implicit constant in $\mathcal{O}\left(\left\|f_{n}\right\|_{n}\right)$ is independent of $\left(f_{n}\right)_{n}$. Putting things together, we have

$$
\begin{aligned}
\int_{-\theta_{n}}^{\theta_{n}} f_{n}\left(x \mathrm{e}^{\mathrm{i} \theta}\right) \exp \left(g_{n}(\theta)\right) \mathrm{d} \theta= & f_{n}(x) \int_{-\theta_{n}}^{\theta_{n}} \mathrm{e}^{-\frac{\lambda_{2, n} \theta^{2}}{2}}\left(1+\mathcal{O}\left(\lambda_{3, n}^{2} \theta^{6}+\lambda_{4, n} \theta^{4}\right)\right) \mathrm{d} \theta \\
& +f_{n}(x) \int_{-\theta_{n}}^{\theta_{n}} \mathrm{e}^{-\frac{\lambda_{2, n} \theta^{2}}{2}} \mathcal{O}\left(\left\|f_{n}\right\|_{n}\right) \mathrm{d} \theta .
\end{aligned}
$$

By (3.9), $\lambda_{2, n} \theta_{n}^{2} \approx n^{1 / 6} \alpha^{-1 / 6}$, which diverges as $n \rightarrow \infty$. The standard estimate on Gaussian tails gives that for all $m \in \mathbb{N}$,

$$
\int_{-\theta_{n}}^{\theta_{n}} \mathrm{e}^{-\frac{\lambda_{2, n} \theta^{2}}{2}} \mathrm{~d} \theta=\int_{-\infty}^{\infty} \mathrm{e}^{-\frac{\lambda_{2, n} \theta^{2}}{2}} \mathrm{~d} \theta+\mathcal{O}\left(\lambda_{2, n}^{-m}\right)=\frac{\sqrt{2 \pi}}{\sqrt{\lambda_{2, n}}}+\mathcal{O}\left(\lambda_{2, n}^{-m}\right) .
$$

A scaling argument, (3.7) and assumption (3.9) give

$$
\int_{-\theta_{n}}^{\theta_{n}} \mathrm{e}^{-\frac{\lambda_{2, n} \theta^{2}}{2}} \lambda_{3, n}^{2}|\theta|^{6} \mathrm{~d} \theta \leq 15 \frac{\sqrt{2 \pi}}{\sqrt{\lambda_{2, n}}} \frac{\lambda_{3, n}^{2}}{\lambda_{2, n}^{3}}=\frac{\sqrt{2 \pi}}{\sqrt{\lambda_{2, n}}} \mathcal{O}\left(\frac{\alpha}{n}\right)
$$

and

$$
\int_{-\theta_{n}}^{\theta_{n}} \mathrm{e}^{-\frac{\lambda_{2, n} \theta^{2}}{2}} \lambda_{4, n}|\theta|^{4} \mathrm{~d} \theta \leq 3 \frac{\sqrt{2 \pi}}{\sqrt{\lambda_{2, n}}} \frac{\lambda_{4, n}}{\lambda_{2, n}^{2}}=\frac{\sqrt{2 \pi}}{\sqrt{\lambda_{2, n}}} \mathcal{O}\left(\frac{\alpha}{n}\right) .
$$


Altogether, we find that

$$
\int_{-\theta_{n}}^{\theta_{n}} f_{n}\left(x \mathrm{e}^{\mathrm{i} \theta}\right) \exp \left(g_{n}(\theta)\right) \mathrm{d} \theta=f_{n}(x) \sqrt{\frac{2 \pi}{\lambda_{2, n}}}\left(1+\mathcal{O}\left(\frac{\alpha}{n}\right)\right)\left(1+\mathcal{O}\left(\left\|f_{n}\right\|_{n}\right) .\right.
$$

What remains to be shown is that

$$
\int_{|\theta| \geq \theta_{n}} f_{n}\left(x \mathrm{e}^{\mathrm{i} \theta}\right) \exp \left(g_{n}(\theta)\right) \mathrm{d} \theta=\mathcal{O}\left(f_{n}(x) \frac{\alpha}{n \sqrt{\lambda_{2, n}}}\right),
$$

where the implicit error term only depends on $\left(f_{n}\right)_{n}$ via $K, N$. We have $-\Re g_{n}(\theta)=\sum_{j=1}^{\alpha} \frac{q_{j, n}}{j}(1-$ $\cos (j \theta)) x^{j}$. For $\theta_{n} \leq \theta<\pi / \alpha$, due to $-\partial_{\theta} \Re g_{n}(\theta)>0$, we have

$$
-\Re g_{n}(\theta) \geq-\Re g_{n}\left(\theta_{n}\right) \approx \theta_{n}^{2} \lambda_{2, n} \approx\left(\frac{n}{\alpha}\right)^{1 / 6}
$$

by assumption $(3.9)$. For $\theta>\frac{\pi}{\alpha}$, let us first assume that $q_{j, n} \geq c>0$ for all $n$ and $j$, i.e. $b(n)=1$ in assumption (iii). We use that

$$
-\Re g_{n}(\theta)=\sum_{j=1}^{\alpha} \frac{q_{j, n}}{j}(1-\cos (j \theta)) x^{j} \geq \frac{c}{\alpha} \sum_{j=1}^{\alpha}(1-\cos (j \theta)) x^{j}=: c r_{n}(\theta)
$$

and

$$
r_{n}(\theta)=\frac{1}{\alpha}\left(x \frac{x^{\alpha}-1}{x-1}-\Re\left(x \mathrm{e}^{\mathrm{i} \theta} \frac{x^{\alpha} \mathrm{e}^{\mathrm{i} \theta \alpha}-1}{x \mathrm{e}^{\mathrm{i} \theta}-1}\right)\right) \geq \frac{2}{\pi^{2}} \frac{x^{\alpha+1}}{\alpha(x-1)} \frac{\theta^{2}}{(x-1)^{2}+\theta^{2}}-\frac{2 x}{\alpha(x-1)} .
$$

The calculations for the final inequality can e.g. be found in [21, Lemma 12]. By (3.8), there exist $c_{1}, c_{2}>0$ with $c_{1} \log \frac{n}{\alpha} \leq \alpha \log x \leq c_{2} \log \frac{n}{\alpha}$. Thus $x \sim 1$, and $x-1 \sim \log x \geq \frac{c_{1}}{\alpha} \log \frac{n}{\alpha}$. So the second term on the right hand side of 3.19 converges to zero. For the first term, we use that $\theta^{2} /\left((x-1)^{2}+\theta^{2}\right)$ is monotone increasing in $\theta$, and find an asymptotic lower bound of the form

$$
\frac{2}{\pi^{2}} \frac{x^{\alpha+1}}{c_{2} \log \frac{n}{\alpha}} \frac{\pi^{2} \alpha^{-2}}{c_{2}^{2} \alpha^{-2}\left(\log \frac{n}{\alpha}\right)^{2}+\pi^{2} \alpha^{-2}} \sim \frac{2}{c_{2}^{3}} \frac{x^{\alpha+1}}{\left(\log \frac{n}{\alpha}\right)^{3}} .
$$

Since $x^{\alpha} \geq\left(\frac{n}{\alpha}\right)^{c_{1}}$, and using condition 3.10 , we conclude that when $\theta \geq \theta_{n}$ and $n \geq N$, $\left|f_{n}\left(x \mathrm{e}^{\mathrm{i} \theta}\right) \mathrm{e}^{g_{n}(\theta)}\right| \leq n^{K}\left|\mathrm{e}^{g_{n}(\theta)}\right|$ vanishes faster than all powers of $1 / n$. This shows the claim in the case $b(n)=1$. For the case of general $b(n)$, we have

$$
\begin{aligned}
-\Re g_{n}(\theta) & \geq \frac{1}{\alpha} \sum_{j=1}^{\alpha} q_{j, n}(1-\cos (\theta j)) x^{j}=c r_{n}(\theta)+\frac{1}{\alpha} \sum_{j=1}^{\alpha}\left(q_{j, n}-c\right)(1-\cos (\theta j)) x^{j} \\
& \geq c r_{n}(\theta)-\frac{2 c}{\alpha} \sum_{j=1}^{b(n)} x^{j} \geq c r_{n}(\theta)\left(1-\frac{2 b(n)}{r_{n}(\theta) \alpha} x^{b(n)}\right) .
\end{aligned}
$$

By assumption, $b(n) / \alpha \leq 1-\delta$ for some $\delta>0$, and then $x^{b(n)-\alpha} \leq\left(\frac{n}{\alpha}\right)^{c_{1} \frac{b(n)-\alpha}{\alpha}} \leq\left(\frac{n}{\alpha}\right)^{-c_{1} \delta}$. Thus, by applying (3.20), the bracket on the right hand side of (3.21) converges to 1 as $n \rightarrow \infty$, and the proof is finished.

\section{Proofs of the MAIN ReSUlts}

We establish most of our results by computing moment generating functions. In the cases we consider, it is a consequence of [28] that pointwise convergence of the moment generating functions in the sector $\left(\mathbb{R}_{0}^{+}\right)^{d}$ is sufficient to establish convergence in distribution of $d$-dimensional random variables. The first result shows that the triangular array $\boldsymbol{q}$ with $q_{j, n}=\mathbb{1}_{\{j \leq \alpha(n)\}}$ is admissible.

Lemma 4.1. Let $x_{n, \alpha}$ be defined by equation (2.1). We have, as $n \rightarrow \infty$ :

$$
\alpha(n) \log \left(x_{n, \alpha}\right)=\log \left(\frac{n}{\alpha(n)} \log \left(\frac{n}{\alpha(n)}\right)\right)+\mathcal{O}\left(\frac{\log (\log (n))}{\log (n)}\right) .
$$


In particular, $x_{n, \alpha} \geq 1, \lim _{n \rightarrow \infty} x_{n, \alpha}=1$ and $x_{n, \alpha}^{\alpha(n)} \sim \frac{n}{\alpha(n)} \log \left(\frac{n}{\alpha(n)}\right)$. Furthermore,

$$
\sum_{j=1}^{\alpha(n)} j x_{n, \alpha}^{j} \sim n \alpha(n)
$$

The first part of the lemma is a reformulation of Lemma 4.11 in [6], which in turn follows [21]. In the latter reference, the claims are actually shown for more general functions $\alpha$. Equation (4.2) has been proved in Lemma 9 in [21. It may also be derived as a special case of Lemma 4.5.

4.1. Proof of Proposition 2.1. Equation (2.3) follows directly from Lemma 4.1. We apply equation (3.4) with $K=1$, differentiate with respect to $s_{1}$, set $s_{1}=0$ and obtain

$$
\mathbb{E}_{n, \alpha}\left[C_{m(n)}\right]=\frac{1}{Z_{n, \alpha}}\left[z^{n}\right] \frac{z^{m(n)}}{m(n)} \exp \left(\sum_{j=1}^{\alpha(n)} \frac{z^{j}}{j}\right) \text {. }
$$

We may now apply Proposition 3.2 with $f_{n}(z)=\frac{z^{m(n)}}{m(n)}$ and $q_{j, n}=\mathbb{1}_{\{j \leq \alpha(n)\}}$. The array $\boldsymbol{q}$ is admissible by Lemma 4.1 and $m(n) \leq \alpha(n)=o\left(\theta_{n}^{-1}\right)$ shows admissibility of $\left(f_{n}\right)$. The claim then follows from $\mathbb{E}_{n, \alpha}\left[C_{m(n)}\right] \sim f_{n}\left(x_{n, \alpha}\right)$.

4.2. Proof of Theorem 2.2. We follow the ideas in [3], where the case of uniform permutations is treated. Let $\left(Z_{k}\right)_{k}$ be independent random variables with $Z_{k} \sim \operatorname{Poi}\left(\frac{1}{k}\right)$ for $k \in \mathbb{N}$ and let

$$
T_{b_{1} b_{2}}:=\sum_{k=b_{1}+1}^{b_{2}} k Z_{k}
$$

Let $\boldsymbol{C}_{b}=\left(C_{1}, C_{2}, \ldots, C_{b}\right)$ the vector of the cycle counts up to length $b, Z_{b}=\left(Z_{1}, Z_{2}, \ldots, Z_{b}\right)$, and $\boldsymbol{a}=\left(a_{1}, a_{2}, \ldots, a_{b}\right)$ a vector. A corner stone for investigating the classical case of uniform random permutations is the so-called conditioning relation [2, Equation (1.15)],

$$
\mathbb{P}_{n}\left[\boldsymbol{C}_{b}=\boldsymbol{a}\right]=\mathbb{P}\left[\boldsymbol{Z}_{b}=\boldsymbol{a} \mid T_{0 n}=n\right] .
$$

Since $\mathbb{P}_{n, \alpha}=\mathbb{P}_{n}\left[\cdot \mid C_{\alpha(n)+1}=\ldots=C_{n}=0\right]$, an analogue of Equation 4.4 holds for $b \leq \alpha(n)$ :

$$
\mathbb{P}_{n, \alpha}\left[\boldsymbol{C}_{b}=\boldsymbol{a}\right]=\mathbb{P}\left[\boldsymbol{Z}_{b}=\boldsymbol{a} \mid T_{0 \alpha(n)}=n\right]
$$

Let $L(\boldsymbol{a}):=\sum_{k=1}^{b(n)} k a_{k}$. For $\boldsymbol{a} \in \mathbb{N}^{b(n)}$ with $L(\boldsymbol{a})=r$, independence of the $Z_{k}$ gives

$$
\mathbb{P}\left[\boldsymbol{Z}_{b(n)}=\boldsymbol{a} \mid T_{0 \alpha(n)}=n\right]=\frac{\mathbb{P}\left[\boldsymbol{Z}_{b(n)}=\boldsymbol{a}\right] \mathbb{P}\left[T_{b(n) \alpha(n)}=n-r\right]}{\mathbb{P}\left[T_{0 \alpha(n)}=n\right]}
$$

Define $\mathbb{P}_{n, b(n), \alpha}$ and $\tilde{\mathbb{P}}_{b(n)}$ as in Theorem 2.2, and let $d_{b(n)}:=\left\|\mathbb{P}_{n, b(n), \alpha}-\tilde{\mathbb{P}}_{b(n)}\right\|_{\mathrm{TV}}$. By 4.4 ,

$$
\begin{aligned}
d_{b(n)} & =\sum_{r=0}^{\infty} \sum_{\boldsymbol{a}: L(\boldsymbol{a})=r} \mathbb{P}\left[\boldsymbol{Z}_{b(n)}=\boldsymbol{a}\right]\left(1-\frac{\mathbb{P}\left[T_{b(n) \alpha(n)}=n-r\right]}{\mathbb{P}\left[T_{0 \alpha(n)}=n\right]}\right)_{+} \\
& =\sum_{r=0}^{\infty} \mathbb{P}\left[T_{0 b(n)}=r\right]\left(1-\frac{\mathbb{P}\left[T_{b(n) \alpha(n)}=n-r\right]}{\mathbb{P}\left[T_{0 \alpha(n)}=n\right]}\right)_{+} \\
& \leq \mathbb{P}\left[T_{0 b(n)} \geq \rho_{n} b(n)+1\right]+\sum_{r=0}^{\rho_{n} b(n)} \mathbb{P}\left[T_{0 b(n)}=r\right]\left(1-\frac{\mathbb{P}\left[T_{b(n) \alpha(n)}=n-r\right]}{\mathbb{P}\left[T_{0 \alpha(n)}=n\right]}\right)_{+},
\end{aligned}
$$

where $\rho_{n}>0$ is arbitrary for now. In [3, Lemma 8] it is shown that

$$
\mathbb{P}\left[T_{0 b(n)} \geq \rho_{n} b(n)\right] \leq\left(\frac{\rho_{n}}{\mathrm{e}}\right)^{-\rho_{n}} .
$$

So $\mathbb{P}\left[T_{0 b(n)} \geq \log (n) b(n)\right]$ decays faster than any power of $n$. The proof is then concluded by plugging $\rho_{n}=\log n$ into the estimate of the lemma below. 
Lemma 4.2. Let $(b(n))_{n \in \mathbb{N}}$ be an integer sequence with $b(n)=o\left(\frac{\alpha(n)}{\log (n)}\right)$ and $\rho_{n}=\mathcal{O}(\log (n))$. We then have

$$
\max _{1 \leq r \leq \rho_{n} b(n)}\left(1-\frac{\mathbb{P}\left[T_{b(n) \alpha(n)}=n-r\right]}{\mathbb{P}\left[T_{0 \alpha(n)}=n\right]}\right)_{+}=\mathcal{O}\left(\frac{\alpha(n)}{n}+\frac{b(n)}{\alpha(n)} \log (n)\right)
$$

as $n \rightarrow \infty$.

Proof. We have $\mathbb{E}\left[z^{T_{b_{1} b_{2}}}\right]=\exp \left(\sum_{j=b_{1}+1}^{b_{2}} \frac{z^{j}-1}{j}\right)$. Therefore,

$$
\mathbb{P}\left[T_{b(n) \alpha(n)}=n-r\right]=\left[z^{n-r}\right] \mathrm{e}^{\sum_{j=b(n)+1}^{\alpha(n)} \frac{z^{j}-1}{j}}=\mathrm{e}^{-\sum_{j=b(n)+1}^{\alpha(n)} \frac{1}{j}}\left[z^{n}\right] z^{r} \mathrm{e}^{\sum_{j=b(n)+1}^{\alpha(n)} \frac{z^{j}}{j}}
$$

and

$$
\mathbb{P}\left[T_{0 \alpha(n)}=n\right]=\mathrm{e}^{-\sum_{j=b(n)+1}^{\alpha(n)} \frac{1}{j}}\left[z^{n}\right] \mathrm{e}^{\sum_{j=1}^{b(n)} \frac{z^{j}-1}{j}} \mathrm{e}^{\sum_{j=b(n)+1}^{\alpha(n)} \frac{z^{j}}{j}} .
$$

Since the factors $\exp \left(-\sum_{j=b(n)+1}^{\alpha(n)} \frac{1}{j}\right)$ will cancel in the quotient of the two terms, we see that we are in the situation of Proposition 3.2. We have $q_{j, n}=\mathbb{1}_{\{b(n)<j \leq \alpha(n)\}}$ in both 4.6 and (4.7). Thus, the relevant saddle point $x_{n, b, \alpha}$ is the unique positive solution of $n=\sum_{j=b(n)+1}^{\alpha(n)} x_{n, b, \alpha}^{j}$. With $x_{n, \alpha}:=x_{n, 0, \alpha}$ defined by 2.1, we easily see that $x_{n, \alpha} \leq x_{n, b, \alpha} \leq x_{n, \frac{\alpha}{2}}$ for large $n$. So Lemma 4.1 shows $\alpha \log x_{n, b, \alpha} \approx \log \frac{n}{\alpha(n)}$ and $\lambda_{2, n} \approx n \alpha(n)$. Thus $\boldsymbol{q}$ is admissible.

In (4.6), we have $f_{n}(z)=f^{(r)}(z)=z^{r}$ for all $n$ in the context of Proposition 3.2. Then, $f^{(r)}$ fulfils (3.10) with $N=K=1$ for all $r \in \mathbb{N}$, and $\left\|f^{(r)}\right\|_{n} \leq r \theta_{n}=\mathcal{O}\left(\theta_{n} b(n) \log (n)\right)$ uniformly in $r \leq \rho_{n} b(n)$. By the assumption on $(b(n)), f^{(r)}$ is $(\delta, 1,1)$-admissible for all $\delta>0$.

In (4.7), $f_{n}(z)=f_{b, n}(z)=\exp \left(\sum_{j=1}^{b(n)} \frac{z^{j}-1}{j}\right)$. We have $\left\|f_{b, n}\right\|_{n} \leq \theta_{n} \sum_{j=0}^{b(n)-1} x_{n, b, \alpha}^{j} \leq \theta_{n} b(n) x_{n, b, \alpha}^{b(n)}$ and

$$
b(n) \log x_{n, b, \alpha} \approx \frac{b(n)}{\alpha(n)} \log \left(\frac{n}{\alpha(n)}\right)=o(1)
$$

by the assumptions on $(b(n))$. Thus, $\left(f_{b, n}\right)_{n}$ is admissible. We conclude

$$
\frac{\mathbb{P}\left[T_{b(n) \alpha(n)}=n-r\right]}{\mathbb{P}\left[T_{0 \alpha(n)}=n\right]}=\frac{f_{r}\left(x_{n, b, \alpha}\right)}{f_{b, n}\left(x_{n, b, \alpha}\right)}\left(1+\mathcal{O}\left(\frac{\alpha(n)}{n}+\theta_{n} b(n) \log n\right)\right),
$$

uniformly in $1 \leq r \leq \rho_{n} b(n)$. Now, $f^{(r)}\left(x_{n, b, \alpha}\right) \geq 1$ since $x_{n, b, \alpha} \geq 1$. On the other hand, we find

$$
0 \leq \log \left(f_{b, n}\left(x_{n, b, \alpha}\right)\right)=\sum_{j=1}^{b(n)} \frac{\left(x_{n, b, \alpha}\right)^{j}-1}{j}=\int_{1}^{x_{n, b, \alpha}} \sum_{j=0}^{b(n)-1} v^{j} \mathrm{~d} v \leq\left(x_{n, b, \alpha}-1\right) b(n)\left(x_{n, b, \alpha}\right)^{b(n)}
$$

By 4.8$),\left(x_{n, b, \alpha}\right)^{b(n)}=\mathcal{O}(1)$, and so $\left(x_{n, b, \alpha}-1\right) b(n)\left(x_{n, b, \alpha}\right)^{b(n)}=\mathcal{O}\left(\frac{b(n)}{\alpha(n)} \log n\right)$. We conclude that $1 \leq f_{b, n}(x) \leq 1+\mathcal{O}\left(\frac{b(n)}{\alpha(n)} \log n\right)$. Hence,

$$
\frac{f_{r}(x)}{f_{b, n}(x)} \geq 1+\mathcal{O}\left(\frac{b(n)}{\alpha(n)} \log n\right) .
$$

The claim now follows by inserting this into 4.9 . 
4.3. Proof of Theorem 2.3. Write $\mu_{j}:=\mu_{m_{j}}$ and $\tilde{C}_{m_{j}}:=C_{m_{j}}^{\left(\mu_{j}\right)}$. Let $s_{j} \geq 0$. We have

$$
\begin{aligned}
& \mathbb{E}\left[\exp \left(\sum_{j=1}^{k} s_{j} \tilde{C}_{m_{j}}\right)\right]=\sum_{l_{1}=0}^{\infty} \cdots \sum_{l_{k}=0}^{\infty} \exp \left(\sum_{j=1}^{k} s_{j} l_{j}\right) \mathbb{P}\left[\tilde{C}_{m_{1}}=l_{1}, \ldots, \tilde{C}_{m_{k}}=l_{k}\right] \\
= & \frac{1}{Z} \sum_{l_{1}=0}^{\infty} \cdots \sum_{l_{k}=0}^{\infty} \prod_{j=1}^{k} \frac{\exp \left(s_{j} l_{j}+\mu_{j}\right)}{\mu_{j}^{l_{j}}} \mathbb{P}_{n, \alpha}\left[C_{m_{1}}=l_{1}, \ldots, C_{m_{k}}=l_{k}\right] \\
= & \frac{\exp \left(\sum_{j=1}^{k} \mu_{j}\right)}{Z} \sum_{l_{1}=0}^{\infty} \cdots \sum_{l_{k}=0}^{\infty} \prod_{j=1}^{k} \exp \left[l_{j}\left(s_{j}-\log \mu_{j}\right)\right] \mathbb{P}_{n, \alpha}\left[C_{m_{1}}=l_{1}, \ldots, C_{m_{k}}=l_{k}\right] \\
= & \frac{\exp \left(\sum_{j=1}^{k} \mu_{j}\right)}{Z} \mathbb{E}_{n, \alpha}\left[\exp \left(\sum_{j=1}^{k}\left(s_{j}-\log \mu_{j}\right) C_{m_{j}}\right)\right] .
\end{aligned}
$$

Here, the normalization $Z$ depends on $n$. By Equation (3.4), the last expectation is given by $Z_{n, \alpha}^{-1}\left[z^{n}\right] f_{n}(z) \exp \left(\sum_{i=1}^{\alpha(n)} \frac{z^{i}}{i}\right)$, with $f_{n}(z):=\exp \left(\sum_{j=1}^{k}\left(\mathrm{e}^{s_{j}-\log \mu_{j}}-1\right) \frac{z^{m_{j}}}{m_{j}}\right)$. We are thus in the framework of Proposition 3.2, with $q_{j, n}=\mathbb{1}_{\{j \leq \alpha(n)\}}$. By Lemma 4.1, it only remains to check admissibility of $\left(f_{n}\right)$. For 3.10 , note that $\left|f_{n}(z)\right| \leq \exp \left(\sum_{j=1}^{k}\left|\mathrm{e}^{s_{j}-\log \left(\mu_{j}\right)}-1\right| \frac{x_{n, \alpha}^{j}}{j}\right)$ and

$$
\left|\mathrm{e}^{s_{j}-\log \left(\mu_{j}\right)}-1\right|=\left(\mathrm{e}^{s_{j}-\log \left(\mu_{j}\right)}-1\right)+2\left(1-\mathrm{e}^{s_{j}-\log \left(\mu_{j}\right)}\right)_{+} \leq\left(\mathrm{e}^{s_{j}-\log \left(\mu_{j}\right)}-1\right)+2 .
$$

Since $\mu_{j}=\frac{x_{n, \alpha}^{j}}{m_{j}}$ by definition and $K_{0}=\sup \left\{\mu_{j}: n \in \mathbb{N}, j \leq k\right\}<\infty$ by assumption (2.6), we get

$$
\left|f_{n}(z)\right| \leq K_{1} f_{n}\left(x_{n, \alpha}\right)
$$

if $|z|=x_{n, \alpha}$, for all $s_{k} \geq 0$, with $K_{1}=\exp \left(2 k K_{0}\right)$. For computing $\left\|f_{n}\right\|_{n}$, a direct calculation together with 4.10 gives

$$
\left|\frac{f_{n}^{\prime}(z)}{f_{n}\left(x_{n, \alpha}\right)}\right| \leq K_{1} \sum_{j=1}^{k}\left|\mathrm{e}^{s_{j}-\log \left(\mu_{j}\right)}-1\right| x_{n, \alpha}^{m_{j}-1} \leq K_{1} \sum_{j=1}^{k}\left(\frac{\mathrm{e}^{s_{j}}}{\mu_{j}}+1\right) \mu_{j} m_{j} \leq K_{1}\left(\sum_{j=1}^{k} \mathrm{e}^{s_{j}}+k K_{0}\right) \alpha .
$$

So, $\left\|f_{n}\right\|_{n} \leq K_{1}\left(\sum_{j=1}^{k} \mathrm{e}^{s_{j}}+k K_{0}\right) \theta_{n} \alpha(n)=o(1)$, and $\left(f_{n}\right)$ is admissible. By Proposition 3.2 , we obtain

$$
\mathbb{E}\left[\exp \left(\sum_{j=1}^{k} s_{j} \tilde{C}_{m_{j}}\right)\right] \sim \frac{\exp \left(\sum_{j=1}^{k} \mu_{j}\right)}{Z} f_{n}\left(x_{n, \alpha}\right)=\frac{\prod_{j=1}^{k} \exp \left(\mathrm{e}^{s_{j}}\right)}{Z}
$$

By setting $s_{j}=0$ for all $j$, we may deduce $Z \rightarrow \mathrm{e}^{k}$ as $n \rightarrow \infty$, and the claim is proved.

4.4. Proof of Theorem 2.4. We now turn to the case of diverging expectation. The following proposition states the most general result in this regime.

Proposition 4.3. Let $m_{j}: \mathbb{N} \rightarrow \mathbb{N}$ for $1 \leq j \leq k$ such that $m_{j}(n) \leq \alpha(n)$ and $m_{i}(n) \neq m_{j}(n)$ for $i \neq j$. Further, let $\mu_{m_{j}(n)}(n)$ as in 2.2 . If $\mu_{m_{j}(n)}(n) \rightarrow \infty$ and $\theta_{n} \frac{x_{n, \alpha}^{m_{j}(n)}}{\sqrt{\mu_{m_{j}(n)}(n)}} \rightarrow 0$ for all $j$, then

$$
\lim _{n \rightarrow \infty} \mathbb{E}_{n, \alpha}\left[\prod_{j=1}^{k} \exp \left(s_{j} \frac{C_{m_{j}(n)}-\mu_{m_{j}(n)}(n)}{\sqrt{\mu_{m_{j}(n)}(n)}}\right)\right]=\exp \left(\sum_{j=1}^{k} \frac{s_{j}^{2}}{2}\right)
$$

for all $s_{j} \geq 0$ and with $\theta_{n}$ as in 3.11.

Note that Theorem 2.2 in [12 shows that it is sufficient to compute the Laplace transform in Proposition 4.3 for $s_{j} \geq 0$ to prove Theorem 2.4 . 
Proof. Write $\mu_{j}:=\mu_{m_{j}(n)}(n)$. Applying equation 3.4 with $s_{k}$ replaced by $s_{j} / \sqrt{\mu_{j}}$, we are in the framework of Proposition 3.2. Again $q_{i, n}:=\mathbb{1}_{\{i \leq \alpha(n)\}}$, so $\boldsymbol{q}$ is admissible, and

$$
f_{n}(z)=\exp \left[\sum_{j=1}^{k}\left(\exp \left(\frac{s_{j}}{\sqrt{\mu_{j}(n)}}\right)-1\right) \frac{z^{m_{j}(n)}}{m_{j}(n)}\right] \exp \left(-\sum_{j=1}^{k} s_{j} \sqrt{\mu_{j}(n)}\right)
$$

For admissibility of $\left(f_{n}\right)$, we compute

$$
\sup _{z \in \partial B_{x_{n, \alpha}}(0)} \frac{\left|f_{n}^{\prime}(z)\right|}{\left|f_{n}(|z|)\right|} \leq \sum_{j=1}^{k}\left(\exp \left(\frac{s_{j}}{\sqrt{\mu_{j}(n)}}\right)-1\right) x_{n, \alpha}^{m_{j}(n)-1} .
$$

By our assumption on $\mu_{m_{j}}(n),\left(f_{n}\right)$ is admissible and we may apply Proposition 3.2. Again the case $s_{j}=0$ for all $j$ deals with the normalizing constant, and so, from

$$
\begin{aligned}
f_{n}\left(x_{n, \alpha}\right) & =\exp \left[\sum_{j=1}^{k}\left(\frac{s_{j}}{\sqrt{\mu_{j}(n)}}+\frac{s_{j}^{2}}{2 \mu_{j}(n)}+\mathcal{O}\left(\frac{s_{j}^{3}}{\left(\mu_{j}(n)\right)^{\frac{3}{2}}}\right)\right) \mu_{j}(n)-\sum_{j=1}^{k} s_{j} \sqrt{\mu_{j}(n)}\right] \\
& =\exp \left[\sum_{j=1}^{k} \frac{s_{j}^{2}}{2}\right]\left(1+\mathcal{O}\left(\sum_{j=1}^{k} \frac{1}{\left(\mu_{j}(n)\right)^{\frac{1}{2}}}\right)\right) \rightarrow \exp \left[\sum_{j=1}^{k} \frac{s_{j}^{2}}{2}\right],
\end{aligned}
$$

we then conclude the claim.

Proof of Theorem 2.4. We check that we can apply Proposition 4.3 . We have $\mu_{m_{j}(n)}(n) \rightarrow \infty$ by assumption. Furthermore, we get with the definition of $\mu_{j}$ in $(2.2)$ and Lemma 4.1 that

$$
\frac{x_{n, \alpha}^{m_{j}(n)}}{\sqrt{\mu_{j}(n)}}=\sqrt{m_{j}(n) x_{n, \alpha}^{m_{j}(n)}} \leq \sqrt{\alpha(n) x_{n, \alpha}^{\alpha(n)}}=\mathcal{O}\left(\sqrt{n \log \left(\frac{n}{\alpha(n)}\right)}\right) .
$$

Using the definition of $\theta_{n}$ in 3.11, we obtain

$$
\theta_{n} \frac{x_{n, \alpha}^{m_{j}(n)}}{\sqrt{\mu_{j}(n)}}=n^{-\frac{5}{12}} \alpha(n)^{-\frac{7}{12}} \frac{x_{n, \alpha}^{m_{j}(n)}}{\sqrt{\mu_{j}(n)}}=\mathcal{O}\left(n^{\frac{1}{12}} \alpha(n)^{-\frac{7}{12}} \log \left(\frac{n}{\alpha(n)}\right)\right)=o(1)
$$

since we assume there is a $\delta>0$ such that $\frac{1}{\alpha(n)}=\mathcal{O}\left(n^{-\frac{1}{7}-\delta}\right)$. The claim is proved.

4.5. Proofs of Theorems 2.5 and 2.6 and Equation 2.10. We begin with equation 3.5, where we plug in $s_{i} / \gamma(n)$ instead of $s_{i}$ for a real-valued sequence $(\gamma(n))_{n \in \mathbb{N}}$. In the terms of Proposition 3.2 this means that $f_{n}=1$ and $q_{j, n}=\mathrm{e}^{\sum_{l=i(j)}^{m} \frac{s_{l}}{\gamma(n)}}$ where $i(j):=\min \left\{1 \leq l \leq m: b_{t_{l}}(n) \geq j\right\}$. Intuitively, any index $l$ with $b_{t_{l}}(n) \geq j$ contributes a factor of $\exp \left(s_{l} / \gamma(n)\right)$ to $q_{j, n}$ since the number of cycles of length $j$ is counted in $K_{b_{t_{l}}}(n)$ in this case. The saddle point of this problem is given by the unique positive solution $x_{n}(s):=x_{n, \alpha, \gamma, t}(s)$ of

$$
n=\sum_{i=0}^{m} \mathrm{e}^{\sum_{l=i+1}^{m} \frac{s_{l}}{\gamma(n)}} \sum_{j=b_{t_{i}}(n)+1}^{b_{t_{i+1}}(n)}\left(x_{n}(s)\right)^{j} .
$$

Note that $x_{n}(\mathbf{0})=x_{n, \alpha}$. Lemmata 4.4 and 4.5 show that $\boldsymbol{q}$ is admissible and provide detailed information which will be useful for investigating the moment generating function more closely.

Lemma 4.4. Let $\gamma(n) \rightarrow \infty$ with $\gamma(n) \geq \log (n)$ and $\boldsymbol{t}=\left(t_{i}\right)_{1 \leq i \leq m}$ with $0=t_{0} \leq t_{1}<\ldots<t_{i}<$ $\ldots<t_{m} \leq t_{m+1}=1$ and $s_{i} \geq 0$ for all $1 \leq i \leq m$. Then

$$
\alpha(n) \log \left(x_{n}(s)\right)=\log \left(\frac{n}{\alpha(n)}\right)+\mathcal{O}\left(\frac{\log (\log (n))}{\log (n)}\right)
$$

locally uniformly in $\boldsymbol{s}$. In particular, $\lim _{n \rightarrow \infty} x_{n}(\boldsymbol{s})=1$ locally uniformly in $\boldsymbol{s}$.

Proof. Let $\hat{x}_{n}(\boldsymbol{s})$ be the unique positive solution of $n \exp \left(-\sum_{i=1}^{m} \frac{s_{i}}{\gamma(n)}\right)=\sum_{j=1}^{\alpha(n)}\left(\hat{x}_{n}(\boldsymbol{s})\right)^{j}$. Since $s_{i} \geq 0$ for all $i$, comparing equations 2.1 and 4.11 yields

$$
\hat{x}_{n}(s) \leq x_{n}(s) \leq x_{n, \alpha(n)} .
$$


By a slightly more general version of Lemma 4.1 (cf. [21, Lemma 9]), we also have

$$
\begin{aligned}
\alpha(n) \log \left(\hat{x}_{n}(s)\right) & =\log \left(\frac{n \exp \left(-\sum_{i=1}^{m} \frac{s_{i}}{\gamma(n)}\right)}{\alpha(n)}\right)+\mathcal{O}\left(\frac{\log (\log (n))}{\log (n)}\right) \\
& =\log \left(\frac{n}{\alpha(n)}\right)+\mathcal{O}\left(\frac{\log (\log (n))}{\log (n)}\right)
\end{aligned}
$$

locally uniformly in $s$ due to $\gamma(n) \rightarrow \infty$. Equation 4.12 then follows from 4.13 together with Lemma 4.1 and equation 4.14.

Lemma 4.5. Let $\gamma(n) \rightarrow \infty$ with $\gamma(n) \geq \log (n)$ and $\boldsymbol{t}=\left(t_{1}, \ldots, t_{m}\right)^{T}$ with $0 \leq t_{1}<\ldots<t_{m} \leq 1$ for $m \in \mathbb{N}$. Then, locally uniformly in $s=\left(s_{1}, \ldots, s_{m}\right)^{T} \in[0, \infty)^{m}$,

$$
\lambda_{2, n}=n \alpha(n)+\mathcal{O}\left(\frac{n \alpha(n)}{\log (n)}\right) .
$$

Proof. W.l.o.g., let $0<t_{1}<1$ and $m=1$. As the following calculations will show, larger values of $m$ pose no particular problem since they only produce additional terms of similar structure and $b_{t_{k}}(n) \sim \alpha(n)$ for all $k \geq 1$ in this case. Moreover, let $x:=x_{n, \alpha, \gamma, t}(s)$. Then, using that $\gamma(n) \geq \log (n)$, we obtain

$$
\begin{aligned}
\lambda_{2, n} & =\mathrm{e}^{\frac{s_{1}}{\gamma(n)}} \sum_{j=1}^{b_{t_{1}}(n)} j x^{j}+\sum_{j=b_{t_{1}}(n)+1}^{\alpha(n)} j x^{j}=\left(\sum_{j=1}^{\alpha(n)} j x^{j}\right)\left(1+\mathcal{O}\left(\frac{1}{\log (n)}\right)\right) \\
& =\left(\alpha(n) \frac{x^{\alpha(n)+1}}{x-1}-\frac{x\left(x^{\alpha(n)}-1\right)}{(x-1)^{2}}\right)\left(1+\mathcal{O}\left(\frac{1}{\log (n)}\right)\right) .
\end{aligned}
$$

Since $x \rightarrow 1$ as $n \rightarrow \infty$, we have $x-1=\log (x)+\mathcal{O}\left((x-1)^{2}\right)$. Using this together with Lemma 4.4 completes the proof.

Having proved that $\boldsymbol{q}$ is admissible, Proposition 3.2 yields, for $\gamma(n) \geq \log (n), \boldsymbol{t}=\left(t_{1}, \ldots, t_{m}\right)^{T}$ and fixed $s=\left(s_{1}, \ldots, s_{m}\right)^{T} \in[0, \infty)^{m}$,

$$
M_{n, \gamma}(s):=\mathbb{E}_{n, \alpha}\left[\exp \left(\sum_{i=1}^{m} \frac{s_{i}}{\gamma(n)} K_{b_{t_{i}}(n)}\right)\right]=\frac{1}{Z_{n, \alpha}} \frac{1}{\sqrt{2 \pi n \alpha(n)}} \exp \left[h_{n}(s)\right](1+o(1)),
$$

where $Z_{n, \alpha}$ is the normalizing constant in 3.3 such that $M_{n, \gamma}(\mathbf{0})=1$ and

$$
h_{n}(\boldsymbol{s}):=h_{n, \alpha, \gamma, \boldsymbol{t}}(\boldsymbol{s}):=\sum_{i=0}^{m} \mathrm{e}^{\sum_{l=i+1}^{m} \frac{s_{l}}{\gamma(n)}} \sum_{j=b_{t_{i}}(n)+1}^{b_{t_{i+1}}(n)} \frac{\left(x_{n, \alpha, \gamma, \boldsymbol{t}}(\boldsymbol{s})\right)^{j}}{j}-n \log \left(x_{n, \alpha, \gamma, \boldsymbol{t}}(\boldsymbol{s})\right) .
$$

The next step is to extract more information by investigating the functions $h_{n}$. The proofs will rest on a Taylor expansion of $h_{n}$ about $\mathbf{0}$, so we need expressions and asymptotics for the derivatives of $h_{n}$. We will prove in Section 4.6 for $\gamma(n) \geq \log (n)$ :

(i) $s \mapsto h_{n}(s)$ is infinitely often differentiable,

(ii) $\partial_{s_{i}} h_{n}(\mathbf{0})=\frac{1}{\gamma(n)} \sum_{j=1}^{b_{t_{i}}(n)} \frac{x_{n, \alpha}^{j}}{j}=t_{i} \frac{n}{\gamma(n) \alpha(n)}(1+o(1))$,

(iii) $\partial_{s_{i_{2}}} \partial_{s_{i_{1}}} h_{n}(\mathbf{0})=t_{i_{2}}\left(1-t_{i_{1}}\right) \frac{n}{(\gamma(n))^{2} \alpha(n)}(1+o(1))$ for $i_{2} \leq i_{1}$,

(iv) $\partial_{s_{i_{2}}} \partial_{s_{i_{1}}} h_{n}(s)=\mathcal{O}\left(\frac{n}{(\gamma(n))^{2} \alpha(n)}\right)$ locally uniformly in $s$,

(v) $\partial_{s_{i_{3}}} \partial_{s_{i_{2}}} \partial_{s_{i_{1}}} h_{n}(\boldsymbol{s})=\mathcal{O}\left(\frac{n}{(\gamma(n))^{3} \alpha(n)}\right)$ locally uniformly in $\boldsymbol{s}$.

Due to $M_{n, \gamma}(\mathbf{0})=1$, for fixed $s$ we therefore arrive at

$$
M_{n, \gamma}(\boldsymbol{s})=\exp \left(\nabla h_{n}(\mathbf{0}) \cdot \boldsymbol{s}+\mathcal{O}\left(\frac{n}{\gamma^{2} \alpha}|\boldsymbol{s}|^{2}\right)\right)(1+o(1))
$$

and

$$
M_{n, \gamma}(\boldsymbol{s})=\exp \left(\nabla h_{n}(\mathbf{0}) \cdot \boldsymbol{s}+\frac{1}{2}\left\langle\boldsymbol{s}, H_{h_{n}}(\mathbf{0}) \boldsymbol{s}\right\rangle+\mathcal{O}\left(\frac{n}{\gamma^{3} \alpha}|\boldsymbol{s}|^{3}\right)\right)(1+o(1))
$$


So, by equation 4.16),

$$
\lim _{n \rightarrow \infty} \mathbb{E}_{n, \alpha}\left[\exp \left(\sum_{i=1}^{m} \frac{s_{i}}{n / \alpha(n)} K_{b_{t_{i}}(n)}\right)\right]=\lim _{n \rightarrow \infty} M_{n, \frac{n}{\alpha(n)}}(s)=\exp \left(\sum_{i=1}^{m} s_{i} t_{i}\right),
$$

and, by equation 4.17),

$$
\begin{aligned}
& \lim _{n \rightarrow \infty} \mathbb{E}_{n, \alpha}\left[\exp \left(\sum_{i=1}^{m} \frac{s_{i}}{\sqrt{n / \alpha(n)}}\left(K_{b_{t_{i}}(n)}-\sum_{j=1}^{b_{t_{i}}(n)} \frac{x_{n, \alpha}^{j}}{j}\right)\right)\right] \\
= & \lim _{n \rightarrow \infty} M_{n, \sqrt{n / \alpha(n)}}(\boldsymbol{s}) \exp \left(-\nabla h_{n}(\mathbf{0}) \cdot \boldsymbol{s}\right)=\exp \left(\frac{1}{2}\langle\boldsymbol{s}, A(\boldsymbol{t}) \boldsymbol{s}\rangle\right),
\end{aligned}
$$

where $A(\boldsymbol{t})=\left(A_{i_{1}, i_{2}}\right)$ is symmetric with $A_{i_{1}, i_{2}}=t_{i_{2}}\left(1-t_{i_{1}}\right)$ for $i_{2} \leq i_{1}$. Note that $A(\boldsymbol{t})$ is the covariance matrix of the Brownian bridge. We can now give the

Proof of Theorem 2.5. We apply arguments of the proof of Corollary 3.4 in [13. Let $\epsilon>0$ and choose $0=t_{0}<t_{1}<\ldots<t_{l}=1$ such that $t_{j+1}-t_{j}<\frac{\epsilon}{2}$. Then, due to monotonicity, $\left|\frac{K_{b t(n)}}{n / \alpha(n)}-t\right|>$ $\epsilon$ for some $t \in[0,1]$ implies the existence of an index $j$ such that $\left|\frac{K_{b_{t_{j}}(n)}}{n / \alpha(n)}-t_{j}\right|>\frac{\epsilon}{2}$. Then,

$$
\mathbb{P}_{n, \alpha}\left[\sup _{t \in[0,1]}\left|\frac{K_{b_{t}(n)}}{n / \alpha(n)}-t\right|>\epsilon\right] \leq \sum_{j=1}^{l} \mathbb{P}_{n, \alpha}\left[\left|\frac{K_{b_{t_{j}}(n)}}{n / \alpha(n)}-t_{j}\right|>\frac{\epsilon}{2}\right] \stackrel{n \rightarrow \infty}{\longrightarrow} 0
$$

by equations 4.18 and 4.19.

Equation 4.19) establishes the convergence of the finite-dimensional distributions of the fluctuations to those of the Brownian bridge. In order to show that, under $\mathbb{P}_{n, \alpha}$, the fluctuations $\left(L_{t}(n)\right)_{t \in[0,1]}$ defined in 2.11] converge as a process to the Brownian bridge, we also have to prove tightness. We will apply the criterion that there are $N \in \mathbb{N}, c>0$, and a nondecreasing continuous function $H$ on $[0,1]$ such that

$$
\mathbb{E}_{n, \alpha}\left[\left|L_{t}(n)-L_{t_{1}}(n)\right|^{2}\left|L_{t_{2}}(n)-L_{t}(n)\right|^{2}\right] \leq c\left|H\left(t_{2}\right)-H\left(t_{1}\right)\right|^{2}
$$

for all $0 \leq t_{1} \leq t \leq t_{2} \leq 1$ and all $n \geq N$, which is an instance of [10, Equation (13.14)].

Proposition 4.6. The sequence of processes $\left(L_{t}(n)\right)_{t \in[0,1]}$ under $\mathbb{P}_{n, \alpha}$ is tight in $\mathcal{D}[0,1]$.

In this paper we only prove tightness of $\left(L_{t}(n)\right)_{t \in[\delta, 1]}$ for $0<\delta<1$ since the proof of the general case (in particular suitably generalizing Lemma 4.7 below) is very technical. The main reason for this is that one has to deal with the divergence of $(\log (t))^{\prime}=\frac{1}{t}$ as $t \rightarrow 0$ in the definition of $b_{t}(n)$. The proof of the general statement can be found in [23, Theorem 2.7.5]. We are going to need

Lemma 4.7. Let $0<\delta<1$. Then there are $N \in \mathbb{N}$ and $c>0$ such that

$$
\sum_{j=b_{t_{1}}(n)+1}^{b_{t_{2}}(n)} \frac{x_{n, \alpha}^{j}}{j} \leq c \frac{n}{\alpha(n)}\left(t_{2}-t_{1}\right)
$$

for all $n \geq N$ and $\delta \leq t_{1}<t_{2} \leq 1$ satisfying $b_{t_{2}}(n)-b_{t_{1}}(n) \geq 2$.

Proof. The definition of $b_{t}(n)$ in 2.7 implies that there exists $N_{1}$ such that $b_{\delta}(n) \geq \alpha(n) / 2$ for all $n \geq N_{1}$. Similarly $\delta \leq t_{1}<t_{2}$ implies $b_{\delta}(n) \leq b_{t_{1}}(n)<b_{t_{2}}(n)$. Thus we get

$$
\sum_{j=b_{t_{1}}(n)+1}^{b_{t_{2}}(n)} \frac{x_{n, \alpha}^{j}}{j} \leq \frac{1}{b_{t_{1}}(n)} x_{n, \alpha}^{b_{t_{1}}(n)+1} \sum_{j=0}^{b_{t_{2}}(n)-b_{t_{1}}(n)-1} x_{n, \alpha}^{j} \leq \frac{2}{\alpha(n)} x_{n, \alpha}^{\alpha(n)+1} \frac{x_{n, \alpha}^{b_{t_{2}}(n)-b_{t_{1}}(n)}-1}{x_{n, \alpha}-1} .
$$

Inserting the definition of $b_{t_{1}}(n)$ and $b_{t_{2}}(n)$ then gives

$$
\sum_{j=b_{t_{1}}(n)+1}^{b_{t_{2}}(n)} \frac{x_{n, \alpha}^{j}}{j} \leq \frac{2}{\alpha(n)} x_{n, \alpha}^{\alpha(n)+1} \frac{\exp \left(\log \left(x_{n, \alpha}\right)\left[\frac{\alpha(n)\left(\log \left(t_{2}\right)-\log \left(t_{1}\right)\right)}{\log (n / \alpha(n))}+1\right]\right)-1}{x_{n, \alpha}-1} .
$$


By Lemma $4.1, \log \left(x_{n, \alpha}\right) \frac{\alpha(n)}{\log (n / \alpha(n))} \rightarrow 1$ as $n \rightarrow \infty$. Moreover, $\log \left(t_{2}\right)-\log \left(t_{1}\right) \leq \delta^{-1}\left(t_{2}-t_{1}\right) \leq \delta^{-1}$ and $\alpha(n)(\log (n / \alpha(n)))^{-1}\left(\log \left(t_{2}\right)-\log \left(t_{1}\right)\right) \geq 1$ by assumption. Hence, there are $N \geq N_{1}, c_{1}>0$ such that

$$
\exp \left(\log \left(x_{n, \alpha}\right)\left[\frac{\alpha(n)\left(\log \left(t_{2}\right)-\log \left(t_{1}\right)\right)}{\log (n / \alpha(n))}+1\right]\right) \leq 1+c_{1}\left(\log \left(t_{2}\right)-\log \left(t_{1}\right)\right) \leq 1+\frac{c_{1}}{\delta}\left(t_{2}-t_{1}\right) .
$$

Thus, by Lemma 4.1 .

$$
\sum_{j=b_{t_{1}}(n)+1}^{b_{t_{2}}(n)} \frac{x_{n, \alpha}^{j}}{j} \leq \frac{2 c_{1}}{\delta} \frac{1}{\alpha(n)} \frac{x_{n, \alpha}^{\alpha(n)+1}}{x_{n, \alpha}-1}\left(t_{2}-t_{1}\right) \leq c \frac{n}{\alpha(n)}\left(t_{2}-t_{1}\right)
$$

for some $c>0$.

Proof of Proposition 4.6. We prove equation 4.21) with $H=\mathrm{id}$ for $\delta \leq t_{1} \leq t \leq t_{2}$. By definition,

$$
\begin{aligned}
I_{n} & =\mathbb{E}_{n, \alpha}\left[\left|L_{t}(n)-L_{t_{1}}(n)\right|^{2}\left|L_{t_{2}}(n)-L_{t}(n)\right|^{2}\right] \\
& =\mathbb{E}_{n, \alpha}\left[\left(\frac{K_{b_{t}(n)}-K_{b_{t_{1}}(n)}-\sum_{j=b_{t_{1}}(n)+1}^{b_{t}(n)} \frac{x_{n, \alpha}^{j}}{j}}{\sqrt{n / \alpha(n)}}\right)^{2}\left(\frac{K_{b_{t_{2}}(n)}-K_{b_{t}(n)}-\sum_{j=b_{t}(n)+1}^{b_{t_{2}}(n)} \frac{x_{n, \alpha}^{j}}{j}}{\sqrt{n / \alpha(n)}}\right)^{2}\right] .
\end{aligned}
$$

We only have to deal with $t_{1}, t_{2}$ such that $b_{t_{2}}(n)-b_{t_{1}}(n) \geq 2$ because $I_{n}=0$ otherwise. Consider the moment generating function

$$
\begin{aligned}
F_{n}\left(s_{1}, s_{2}\right): & :=\mathbb{E}_{n, \alpha}\left[\exp \left(s_{1} \frac{K_{b_{t}(n)}-K_{b_{1}(n)}}{\sqrt{n / \alpha(n)}}+s_{2} \frac{K_{b_{t_{2}}(n)}-K_{b_{t}(n)}}{\sqrt{n / \alpha(n)}}\right)\right] \\
& =\frac{1}{Z_{n, \alpha}}\left[z^{n}\right] \exp \left(\sum_{j=1}^{b_{t_{1}}(n)} \frac{z^{j}}{j}+\mathrm{e}^{\sqrt{\frac{\alpha(n)}{n}} s_{1}} \sum_{j=b_{t_{1}}(n)+1}^{b_{t}(n)} \frac{z^{j}}{j}+\mathrm{e}^{\sqrt{\frac{\alpha(n)}{n}} s_{2}} \sum_{j=b_{t}(n)+1}^{b_{t_{2}}(n)} \frac{z^{j}}{j}+\sum_{j=b_{t_{2}}(n)+1}^{\alpha(n)} \frac{z^{j}}{j}\right) .
\end{aligned}
$$

Then $F_{n}$ is differentiable and

$$
\mathbb{E}_{n, \alpha}\left[\left(\frac{K_{b_{t}(n)}-K_{b_{t_{1}}(n)}}{\sqrt{n / \alpha(n)}}\right)^{m_{1}}\left(\frac{K_{b_{t_{2}}(n)}-K_{b_{t}(n)}}{\sqrt{n / \alpha(n)}}\right)^{m_{2}}\right]=\left.\partial_{s_{1}}^{m_{1}} \partial_{s_{2}}^{m_{2}} F_{n}\left(s_{1}, s_{2}\right)\right|_{\left(s_{1}, s_{2}\right)=0}
$$

holds. By linearity of the expectation, we can expand the product in equation 4.22 and then apply Equation 4.23 to each summand. A calculation then yields

$$
I_{n}=\frac{\alpha(n)^{2}}{Z_{n, \alpha} n^{2}}\left[z^{n}\right]\left[G_{n, t_{1}, t}(z) G_{n, t, t_{2}}(z) \exp \left(\sum_{j=1}^{\alpha(n)} \frac{z^{j}}{j}\right)\right]
$$

where

$$
G_{n, t_{1}, t}(z):=\left(\sum_{j=b_{t_{1}}(n)+1}^{b_{t}(n)} \frac{z^{j}-x_{n, \alpha}^{j}}{j}\right)^{2}+\sum_{j=b_{t_{1}}(n)+1}^{b_{t}(n)} \frac{z^{j}}{j}
$$

The additional terms of the form $\sum_{j=b_{t_{1}}(n)+1}^{b_{t}(n)} \frac{z^{j}}{j}$ result from the product rule when calculating the second derivative with respect to the same variable $s_{1}$. We now proceed as in the proof of Proposition 3.2 with $q_{j, n}=\mathbb{1}_{\{j \leq \alpha(n)\}}$, which is admissible. The functions $G_{n, t_{1}, t}(z) G_{n, t, t_{2}}(z)$ would play the role of $f_{n}$, but they only satisfy (i) and (ii) (by Lemma 4.8). Since (iii) does in general not hold, we will have to make some adaptations. As in the proof of Proposition 3.2 , by Cauchy's integral formula, we write $I_{n}$ as a contour integral along $\partial B_{x_{n, \alpha}}(0)$ and introduce the function $g_{n}(\theta)=\sum_{j=1}^{\alpha(n)} x_{n, \alpha}^{j} \frac{\mathrm{e}^{\mathrm{i} j \theta}-1}{j}$. We then arrive at the expression

$$
I_{n}=\frac{\alpha(n)^{2}}{Z_{n, \alpha} n^{2}} \frac{\exp \left(\sum_{j=1}^{\alpha(n)} \frac{x_{n, \alpha}^{j}}{j}\right)}{2 \pi x_{n, \alpha}^{n}} \int_{-\pi}^{\pi} G_{n, t_{1}, t}\left(x_{n, \alpha} \mathrm{e}^{\mathrm{i} \theta}\right) G_{n, t, t_{2}}\left(x_{n, \alpha} \mathrm{e}^{\mathrm{i} \theta}\right) \exp \left(g_{n}(\theta)\right) \mathrm{d} \theta .
$$


We also split the integral into two parts. The main contribution is again due to the interval $\left[-\theta_{n}, \theta_{n}\right]$. By Lemma 4.8 literally retracing the steps in the proof of Proposition 3.2 shows that

$$
\frac{\alpha(n)^{2}}{Z_{n, \alpha} n^{2}} \frac{\exp \left(\sum_{j=1}^{\alpha(n)} \frac{x_{n, \alpha}^{j}}{j}\right)}{2 \pi x_{n, \alpha}^{n}} \int_{\pi \geq|\theta|>\theta_{n}} G_{n, t_{1}, t}\left(x_{n, \alpha} \mathrm{e}^{\mathrm{i} \theta}\right) G_{n, t, t_{2}}\left(x_{n, \alpha} \mathrm{e}^{\mathrm{i} \theta}\right) \exp \left(g_{n}(\theta)\right) \mathrm{d} \theta
$$

vanishes faster than any power of $1 / n$. It poses no problem due to $t_{2}-t_{1} \geq \log (n / \alpha(n)) / \alpha(n)$. For $|\theta| \leq \theta_{n}$, apply $\left|\mathrm{e}^{\mathrm{i} j \theta}-1\right| \leq c_{1} j \theta$ for some $c_{1}>0$ for all $j$ and $\left|\mathrm{e}^{\mathrm{i} j \theta}\right|=1$. Then there is $c_{2}>0$ such that

$$
\begin{aligned}
& \left|G_{n, t_{1}, t}\left(x_{n, \alpha} \mathrm{e}^{\mathrm{i} \theta}\right) G_{n, t, t_{2}}\left(x_{n, \alpha}\right)\right| \\
\leq & c_{2}\left(\left(\theta \sum_{j=b_{t_{1}}(n)+1}^{b_{t}(n)} x_{n, \alpha}^{j}\right)^{2}+\sum_{j=b_{t_{1}}(n)+1}^{b_{t}(n)} \frac{x_{n, \alpha}^{j}}{j}\right)\left(\left(\theta \sum_{j=b_{t}(n)+1}^{b_{t_{2}}(n)} x_{n, \alpha}^{j}\right)^{2}+\sum_{j=b_{t}(n)+1}^{b_{t_{2}}(n)} \frac{x_{n, \alpha}^{j}}{j}\right)
\end{aligned}
$$

for all $n$. Due to equation 3.16), we have $\left|\exp \left(g_{n}(\theta)\right)\right| \leq c_{3} \exp \left(-\frac{\lambda_{2, n}}{2} \theta^{2}\right)$ for some $c_{3}>0$ and all $|\theta| \leq \theta_{n}$ if $n$ is large enough. By substituting $v=\sqrt{\lambda_{2, n}} \theta$, we therefore obtain

$$
\left|\int_{-\theta_{n}}^{\theta_{n}} \theta^{k} \exp \left(g_{n}(\theta)\right) \mathrm{d} \theta\right| \leq c_{4} \lambda_{2, n}^{-\frac{k+1}{2}}
$$

for some $c_{4}>0$ and $0 \leq k \leq 4$ because of the moments of the normal distribution. By linearity of the integral as well as the definition of $Z_{n, \alpha}$ and Lemmata 4.5 and 4.7 we conclude

$$
I_{n} \leq c^{\prime}\left[\left(t-t_{1}\right)^{2}+t-t_{1}\right]\left[\left(t_{2}-t\right)^{2}+\left(t_{2}-t\right)\right] \leq c\left(t_{2}-t_{1}\right)^{2}
$$

for some $c^{\prime}, c>0$ and $n$ large enough. The last step holds due to $\delta \leq t_{1} \leq t \leq t_{2} \leq 1$.

4.6. Properties of $h_{n}$. This section provides the proofs for five properties of $h_{n}$ and its derivatives stated in Section 4.5. We are going to need the asymptotics presented in

Lemma 4.8. Let $0<t \leq 1$. Then,

$$
\sum_{j=1}^{b_{t}(n)} x_{n, \alpha}^{j} \sim \text { tn and } \sum_{j=1}^{b_{t}(n)} \frac{x_{n, \alpha}^{j}}{j} \sim t \frac{n}{\alpha(n)}
$$

hold.

Proof. Since $x_{n, \alpha}>1$, we have

$$
\int_{0}^{b_{t}(n)} x_{n, \alpha}^{v} \mathrm{~d} v \leq \sum_{j=1}^{b_{t}(n)} x_{n, \alpha}^{j} \leq \int_{1}^{b_{t}(n)+1} x_{n, \alpha}^{v} \mathrm{~d} v \sim \int_{0}^{b_{t}(n)} x_{n, \alpha}^{v} \mathrm{~d} v
$$

by Lemma 4.1. It therefore remains to be shown that $\int_{0}^{b_{t}(n)} x_{n, \alpha}^{v} \mathrm{~d} v=\frac{\left(x_{n, \alpha}\right)^{b_{t}(n)}-1}{\log \left(x_{n, \alpha}\right)} \sim t n$. Since $0<\frac{b_{t}(n)}{\alpha(n)} \leq 1$ for $n$ large enough, the first claim follows from equation 4.25 and

$$
\left(x_{n, \alpha}\right)^{b_{t}(n)}=\left[\left(x_{n, \alpha}\right)^{\alpha(n)}\right]^{\frac{b_{t}(n)}{\alpha(n)}} \sim \exp \left[\frac{b_{t}(n)}{\alpha(n)} \log \left(\frac{n}{\alpha(n)} \log \left(\frac{n}{\alpha(n)}\right)\right)\right] \sim t \frac{n}{\alpha(n)} \log \left(\frac{n}{\alpha(n)}\right),
$$

which holds due to Lemma 4.1. It was proved in Proposition 4.8 in [6] that $\sum_{j=1}^{\alpha(n)} \frac{x_{n, \alpha}^{j}}{j} \sim \frac{n}{\alpha(n)}$. Consider $\sum_{j=1}^{b_{t}(n)} \frac{x_{n, \alpha}^{j}}{j}=\sum_{j=1}^{\alpha(n)} \frac{x_{n, \alpha}^{j}}{j}-\sum_{j=b_{t}(n)+1}^{\alpha(n)} \frac{x_{n, \alpha}^{j}}{j}$. Due to $b_{t}(n) \sim \alpha(n)$ and the first claim,

$$
\frac{1}{\alpha(n)} \sum_{j=b_{t}(n)+1}^{\alpha(n)} x_{n, \alpha}^{j} \leq \sum_{j=b_{t}(n)+1}^{\alpha(n)} \frac{x_{n, \alpha}^{j}}{j} \leq \frac{1}{b_{t}(n)+1} \sum_{j=b_{t}(n)+1}^{\alpha(n)} x_{n, \alpha}^{j}
$$

yields the second claim. 
Let $\gamma(n) \geq \log (n), \boldsymbol{t}=\left(t_{1}, \ldots, t_{m}\right)^{T}$ for $m \in \mathbb{N}$ and $h_{n}(\boldsymbol{s})$ as in 4.15 throughout this section. Set further $t_{0}=0$ and $t_{m+1}=1$. Property (i), which states that $h_{n}$ is infinitely often differentiable in $\boldsymbol{s}$, follows from the differentiability of the saddle point $x_{n, \alpha, \gamma, t}$ which can be shown by applying the implicit function theorem to the function $F(s, x)=\sum_{i=0}^{m} \sum_{j=b_{t_{i}}(n)+1}^{b_{t_{i+1}}(n)}\left[\exp \left(\sum_{l=i+1}^{m} \frac{s_{l}}{\gamma(n)}\right) x\right]^{j}-n$, see (4.11). So we can compute the derivatives of $h_{n}$.

Fix $i_{3} \leq i_{2} \leq i_{1}$ and let $x_{n}(\boldsymbol{s}):=x_{n, \alpha, \gamma, \boldsymbol{t}}(\boldsymbol{s})$. For the sake of brevity, we introduce the notations

$$
\lambda_{p, n}^{\left(i_{1}\right)}:=\sum_{i=0}^{i_{1}-1} \mathrm{e}^{\sum_{l=i+1}^{m} \frac{s_{l}}{\gamma(n)}} \sum_{j=b_{t_{i}}(n)+1}^{b_{t_{i+1}}(n)} j^{p-1}\left(x_{n}(s)\right)^{j}
$$

so that $\lambda_{p, n}=\lambda_{p, n}^{(m+1)}$. We obtain

$$
\begin{aligned}
\partial_{s_{i_{1}}} h_{n}(s) & =\frac{1}{\gamma(n)} \lambda_{0, n}^{\left(i_{1}\right)} \\
\partial_{s_{i_{2}}} \partial_{s_{i_{1}}} h_{n}(s) & =\frac{1}{\gamma(n)} \frac{\partial_{s_{i_{2}}} x_{n}(s)}{x_{n}(s)} \lambda_{1, n}^{\left(i_{1}\right)}+\frac{1}{(\gamma(n))^{2}} \lambda_{0, n}^{\left(i_{2}\right)}
\end{aligned}
$$

and

$$
\begin{aligned}
\partial_{s_{i_{3}}} \partial_{s_{i_{2}}} \partial_{s_{i_{1}}} h_{n}(s)= & \frac{1}{(\gamma(n))^{2}} \frac{\partial_{s_{i_{2}}} x_{n}(s)}{x_{n}(s)} \lambda_{1, n}^{\left(i_{3}\right)} \\
& +\frac{1}{\gamma(n)}\left(\frac{\partial_{s_{3}} \partial_{s_{i_{2}}} x_{n}(s)}{x_{n}(s)}-\frac{\partial_{s_{i_{2}}} x_{n}(s)}{x_{n}(\boldsymbol{s})} \frac{\partial_{s_{i_{3}}} x_{n}(\boldsymbol{s})}{x_{n}(\boldsymbol{s})}\right) \lambda_{1, n}^{\left(i_{1}\right)} \\
& +\frac{1}{\gamma(n)} \frac{\partial_{s_{i_{2}}} x_{n}(\boldsymbol{s})}{x_{n}(\boldsymbol{s})} \frac{\partial_{s_{i_{3}}} x_{n}(\boldsymbol{s})}{x_{n}(\boldsymbol{s})} \lambda_{2, n}^{\left(i_{1}\right)}+\frac{1}{(\gamma(n))^{2}} \frac{\partial_{s_{i_{3}}} x_{n}(\boldsymbol{s})}{x_{n}(\boldsymbol{s})} \lambda_{1, n}^{\left(i_{2}\right)}+\frac{1}{(\gamma(n))^{3}} \lambda_{0, n}^{\left(i_{3}\right)} .
\end{aligned}
$$

In order to prove properties (ii) to $(\mathrm{v})$, we need to understand the derivatives of the saddle point.

Lemma 4.9. Fix $i_{2} \leq i_{1}$. Then,

$$
\frac{\partial_{s_{i_{1}}} x_{n}(s)}{x_{n}(s)}=-\frac{1}{\gamma(n)} \frac{\lambda_{1, n}^{\left(i_{1}\right)}}{\lambda_{2, n}}
$$

Moreover,

$$
\frac{\partial_{s_{i_{1}}} x_{n}(s)}{x_{n}(s)}=\mathcal{O}\left(\frac{1}{\gamma(n) \alpha(n)}\right) \text { and } \frac{\partial_{s_{i_{2}}} \partial_{s_{i_{1}}} x_{n}(s)}{x_{n}(s)}-\frac{\partial_{s_{i_{2}}} x_{n}(s)}{x_{n}(s)} \frac{\partial_{s_{i_{1}}} x_{n}(s)}{x_{n}(s)}=\mathcal{O}\left(\frac{1}{(\gamma(n))^{2} \alpha(n)}\right)
$$

hold locally uniformly in $s$.

Proof. Differentiating equation 4.11 with respect to $s_{i_{1}}$ yields $0=\frac{1}{\gamma(n)} \lambda_{1, n}^{\left(i_{1}\right)}+\frac{\partial_{s_{i_{1}}} x_{n}(\boldsymbol{s})}{x_{n}(\boldsymbol{s})} \lambda_{2, n}$, so

$$
\frac{\partial_{s_{i_{1}}} x_{n}(s)}{x_{n}(s)}=-\frac{1}{\gamma(n)} \frac{\lambda_{1, n}^{\left(i_{1}\right)}}{\lambda_{2, n}}=\mathcal{O}\left(\frac{1}{\gamma(n) \alpha(n)}\right)
$$

by equation 4.11 and Lemma 4.5. W.l.o.g., let $i_{2} \leq i_{1}$. Differentiating once more, now with respect to $s_{i_{2}}$, we obtain

$$
\begin{aligned}
\frac{\partial_{s_{i_{2}}} \partial_{s_{i_{1}}} x_{n}(s)}{x_{n}(\boldsymbol{s})}-\frac{\partial_{s_{i_{2}}} x_{n}(\boldsymbol{s})}{x_{n}(\boldsymbol{s})} \frac{\partial_{s_{i_{1}}} x_{n}(\boldsymbol{s})}{x_{n}(\boldsymbol{s})}= & -\frac{1}{(\gamma(n))^{2}} \frac{\lambda_{1, n}^{\left(i_{2}\right)}}{\lambda_{2, n}}-\frac{1}{\gamma(n)} \frac{\partial_{s_{i_{2}}} x_{n}(\boldsymbol{s})}{x_{n}(\boldsymbol{s})} \frac{\lambda_{2, n}^{\left(i_{1}\right)}}{\lambda_{2, n}} \\
& +\frac{1}{(\gamma(n))^{2}} \frac{\lambda_{1, n}^{\left(i_{1}\right)}}{\left(\lambda_{2, n}\right)^{2}} \lambda_{2, n}^{\left(i_{2}\right)}+\frac{1}{\gamma(n)} \frac{\partial_{s_{i_{2}}} x_{n}(s)}{x_{n}(s)} \frac{\lambda_{1, n}^{\left(i_{1}\right)}}{\left(\lambda_{2, n}\right)^{2}} \lambda_{3, n} .
\end{aligned}
$$

Applying Lemma 4.5 equation (3.7), and the first result to each term, we conclude the last claim.

Property (ii) is now a direct consequence of equation 4.26 and Lemma 4.8 (iii) and (iv) follow from equation (4.27) and Lemmata 4.9 and 4.8 . Property (v) can easily be deduced from equation (4.28) and Lemmata 4.9 and 4.8 . 


\section{ACKNOWLEDGEMENTS}

H.S. acknowledges support by Deutsche Telekom Stiftung.

\section{REFERENCES}

[1] R. Arratia, A. D. Barbour, and T. Tavaré. Poisson process approximations for the Ewens sampling formula. Ann. Appl. Probab., 2(3):519-535, 1992.

[2] R. Arratia, A.D. Barbour, and S. Tavaré. Logarithmic combinatorial structures: a probabilistic approach. EMS Monographs in Mathematics. European Mathematical Society (EMS), Zürich, 2003.

[3] R. Arratia and S. Tavaré. The cycle structure of random permutations. The Annals of Probability, pages 1567-1591, 1992.

[4] R. Arratia and S. Tavaré. Limit theorems for combinatorial structures via discrete process approximations. Random Structures Algorithms, 3(3):321-345, 1992.

[5] A. D. Barbour. [poisson approximation and the chen-stein method]: Comment. Statistical Science, 5(4):425427, 111990.

[6] V. Betz and H. Schäfer. The number of cycles in random permutations without long cycles is asymptotically Gaussian. ALEA, 14:427-444, 2017.

[7] V. Betz, D. Ueltschi, Spatial random permutations and infinite cycles, Commun. Math. Phys. 285, 469-501 (2009)

[8] V. Betz, D. Ueltschi, Critical temperature of dilute Bose gases, Phys. Rev. A 81, 023611 (2010)

[9] V. Betz, D. Ueltschi, and Y. Velenik. Random permutations with cycle weights. The Annals of Applied Probability, 21(1):312-331, 2011.

[10] P. Billingsley. Convergence of Probability Measures. Wiley, 1999.

[11] L. V. Bogachev and D. Zeindler. Asymptotic statistics of cycles in surrogate-spatial permutations. Communications in Mathematical Physics, 334(1):39-116, 2015.

[12] P. Chareka A finite-interval uniqueness theorem for bilateral Laplace transforms. Int. J. Math. Math. Sci., 2007, Art. ID 60916, 6

[13] A. Cipriani and D. Zeindler. The limit shape of random permutations with polynomially growing cycle weights. ALEA Lat. Am. J. Probab. Math. Stat., 12(2):971-999, 2015.

[14] J. Delaurentis and B. Pittel. Random permutations and Brownian motion. Pacific Journal of Mathematics, 119(2):287-301, 1985 .

[15] D. Elboim and R. Peled. Limit distributions for Euclidean random permutations arXiv:1712.03809 [math.PR], 2017

[16] N. M. Ercolani and D. Ueltschi. Cycle structure of random permutations with cycle weights. Random Structures Algorithms, 44(1):109-133, 2014.

[17] W.J. Ewens. The sampling theory of selectively neutral alleles. Theoret. Populations Biol., 3:87-112, 1972.

[18] P. Flajolet and R. Sedgewick. Analytic Combinatorics. Cambridge University Press, 2009.

[19] J. F. C. Kingman. The population structure associated with the Ewens sampling formula. Theoret. Population Biology, 11(2):274-283, 1977.

[20] E. Kowalski and A. Nikeghbali. Mod-Poisson convergence in probability and number theory. Int. Math. Res. Not. IMRN, 2010(18):3549-3587, 2010.

[21] E. Manstavičius and R. Petuchovas. Local probabilities for random permutations without long cycles. Electron. J. Combin., 23(1):Paper 1.58, 25, 2016.

[22] G. Pólya. Kombinatorische Anzahlbestimmungen für Gruppen, Graphen, und chemische Verbindungen. Acta Mathematica, 68:145-254, 1937.

[23] H. Schäfer. The Cycle Structure of Random Permutations without Macroscopic Cycles. Doctoral thesis, Technische Universität Darmstadt, 2018. http://tuprints.ulb.tu-darmstadt.de/8148/

[24] A.A. Shmidt and A. M. Vershik. Limit measures arising in the asymptotic theory of symmetric groups. Theory Probab. Appl., 22, No.1:70-85, 1977.

[25] D.Stark, Total variation asymptotics for refined Poisson process approximations of random logarithmic assemblies Combin. Probab. Comput., 8, No.1:567-598, 1999

[26] A. L. Yakymiv. Random A-permutations: convergence to a Poisson process. Mathematical Notes, 81(5-6):840846, 2007.

[27] A. L. Yakymiv. Limit theorem for the general number of cycles in a random A-permutation. Theory of Probability $\mathcal{E}$ Its Applications, 52(1):133-146, 2008.

[28] A. L. Yakymiv. A generalization of the Curtiss theorem for moment generating functions. Mathematical Notes, 90(5):920-924, 2011 